\title{
Seedling and Slow Rusting Resistance to Stripe Rust in Chinese Common Wheats
}

\author{
Z. F. Li and X. C. Xia, Institute of Crop Science/National Wheat Improvement Center, Chinese Academy of Agricul- \\ tural Sciences, Zhongguancun South Street 12, 100081, Beijing, China; X. C. Zhou, Gansu Winter Wheat Research \\ Institute, Duan Jia Tan 418, 730020, Lanzhou, China; Y. C. Niu, Plant Protection Institute, Chinese Academy of Agri- \\ cultural Science, Yuanmingyuan West Road 2, 100094, Beijing, China; Z. H. He, Institute of Crop Science/National \\ Wheat Improvement Center, Chinese Academy of Agricultural Sciences, and International Maize and Wheat Improve- \\ ment Center (CIMMYT) China Office, C/O CAAS, Zhongguancun South Street 12, 100081, Beijing, China; Y. Zhang \\ and G. Q. Li, Institute of Crop Science/National Wheat Improvement Center, Chinese Academy of Agricultural Sci- \\ ences; A. M. Wan, Plant Protection Institute, Chinese Academy of Agricultural Science; D. S. Wang and X. M. Chen, \\ Institute of Crop Science/National Wheat Improvement Center, Chinese Academy of Agricultural Sciences; Q. L. Lu, \\ Gansu Winter Wheat Research Institute; and R. P. Singh, CIMMYT, Apdo. Postal 6-641, 06600, Mexico D.F., Mexico
}

\begin{abstract}
Li, Z. F., Xia, X. C., Zhou, X. C., Niu, Y. C., He, Z. H., Zhang, Y., Li, G. Q., Wan, A. M., Wang, D. S., Chen, X. M., Lu, Q. L., and Singh, R. P. 2006. Seedling and slow rusting resistance to stripe rust in Chinese common wheats. Plant Dis. 90:1302-1312.

Identification of seedling and slow stripe rust resistance genes is important for gene pyramiding, gene deployment, and developing slow-rusting wheat cultivars to control the disease. A total of 98 Chinese lines were inoculated with 26 pathotypes of Puccinia striiformis f. sp. tritici for postulation of stripe rust resistance genes effective at the seedling stage. A total of 135 wheat lines were planted at two locations to characterize their slow rusting responses to stripe rust in the 2003-2004 and 2004-2005 cropping seasons. Genes Yr2, Yr3a, Yr4a, Yr6, Yr7, Yr9, Yr26, $Y r 27$, and $Y r S D$, either singly or in combinations, were postulated in 72 lines, whereas known resistance genes were not identified in the other 26 accessions. The resistance genes $\mathrm{Yr} 9$ and Yr26 were found in 42 and 19 accessions, respectively. Yr3a and $Y r 4 a$ were detected in two lines, and four lines may contain $Y r 6$. Three lines were postulated to possess $Y r S D$, one carried $Y r 27$, and one may possess $Y r 7$. Thirty-three lines showed slow stripe rusting resistance at two locations in both seasons.
\end{abstract}

Additional keywords: bread wheat, gene-for-gene specificity, yellow rust

Stripe rust, caused by Puccinia striiformis $\mathrm{f}$. sp. tritici, is an important disease in many wheat-growing regions of the world. Epidemics of stripe rust have occurred periodically in China and, in some years, have caused destructive yield losses in the major wheat production regions, particularly in northwestern and southwestern China (35). The most recent widespread epidemic, in 2002, was caused by Chinese $P$. striiformis f. sp. tritici pathotype CYR32, which is virulent to almost all Chinese wheat cultivars except those with resistance genes $\mathrm{Yr} 5, \mathrm{Yr} 10, \mathrm{Yr} 15$, Yr24, or $\operatorname{Yr} 26(11,16,35,44)$.

Following the gene-for-gene hypothesis proposed by Flor (7), Zadoks (45) found a gene-for-gene interaction between wheat cultivars and pathotypes of $P$. striiformis $\mathrm{f}$. sp. tritici. Based on the gene-for-gene

Corresponding authors:

X. C. Xia; E-mail: xiaxianchun@ caas.net.cn

Z. H. He; E-mail: zhhe@public3.bta.net.cn

Accepted for publication 1 April 2006.

DOI: 10.1094/PD-90-1302

(C) 2006 The American Phytopathological Society concept, Loegering and Burton (14) reported that race-specific resistance genes in wheat cultivars could be identified by comparing their reactions to different pathotypes of Puccinia graminis tritici with those of lines with known resistance genes. Perwaiz and Johnson (24) tested seedlings of 26 wheat cultivars from Pakistan with 18 British P. striiformis f. sp. tritici pathotypes and postulated $\operatorname{Yr} 2, \operatorname{Yr} 6$, $Y r 7$, and/or $Y r 9$ to be present in the cultivars. Bartos et al. (2) postulated $Y r 1, Y r 2$, $\operatorname{Yr} 3 a+\operatorname{Yr} 4 a, \operatorname{Yr} 9$, and $\operatorname{Yr} 32(\mathrm{YrCV})$ in 17 Czechoslovakian and two Russian wheat cultivars following tests with $18 \mathrm{P}$. striiformis $\mathrm{f}$. sp. tritici pathotypes. Singh et al. (28) found $Y r 2$ and $Y r 7$ in a seedling test of 11 Indian wheat cultivars using 17 pathotypes and confirmed the presence of $Y r 2$ in three cultivars by testing the $\mathrm{F}_{1}$ and $\mathrm{F}_{2}$ generations of crosses between these cultivars and Heines VII, a cultivar that contains $Y r 2$. Sharma et al. (26) inoculated seedlings of 38 wild emmer derivatives and 53 advanced wheat lines from Nepal with $18 P$. striiformis $\mathrm{f}$. sp. tritici pathotypes and found 28 wild emmer derivatives were resistant to all pathotypes with unidentified resistance genes, and five resis- tance genes $(\mathrm{Yr} 2, \mathrm{Yr} 6, \mathrm{Yr} 7, \mathrm{Yr} 9$, and $\mathrm{YrA})$ were present in Nepalese wheat cultivars and advanced lines tested. In China, Wang et al. $(36,37)$ identified eight resistance genes $(\mathrm{Yr} 1, \mathrm{Yr} 2, \mathrm{Yr} 3, \mathrm{Yr} 7, \mathrm{Yr} 9, \mathrm{Yr} 10, \mathrm{YrSu}$, and $Y r S D)$ in 39 Chinese wheat cultivars and 20 important resistant germ plasm lines from the provinces of Shaanxi, Gansu, and Sichuan, using 20 P. striiformis f. sp. tritici pathotypes. Niu et al. (21) screened 50 Chinese wheat cultivars from the provinces of Henan, Shandong, and Anhui with 26 pathotypes, and found that $\operatorname{Yr} 9$ was the most frequent resistance gene. However, no information is available about the identity of stripe rust resistance genes in current leading cultivars, newly released cultivars, and advanced breeding lines in China. Therefore, characterization of stripe rust resistance genes in widely grown Chinese wheats is extremely important for breeding new resistant cultivars and for gene deployment schemes (35).

In general, seedling resistance is racespecific and thus short-lived due to frequent changes of virulence in the pathogen population $(13,31)$. In contrast, resistance at the adult plant stage, such as hightemperature adult-plant (HTAP) resistance or slow rusting, is considered more durable than seedling resistance $(5,6,13,18,32)$. Wheat cultivars with slow rusting genes are often susceptible at the seedling stage, but may be moderately to highly resistant to all pathotypes at the adult plant stage in the field $(4,31)$. The components of slow rusting include a longer latent period, low infection frequency, smaller uredial size, and reduced duration and quantity of spore production (4). Three currently named stripe rust resistance genes, $\operatorname{Yr} 18, \operatorname{Yr} 29$, and $\operatorname{Yr} 30$, confer slow rusting $(29,33,40)$, and many more are suspected. Singh $(29,30)$ and McIntosh (18) found the slow rusting resistance gene $\mathrm{Yrl} 18$ in Anza and Bezostaja, and this gene confers a moderate level of resistance when present alone. However, combinations of $\mathrm{Yr} 18$ with two to four additional slow rusting genes resulted in high resistance in most environments (31). William et al. (40) identified 
slow rusting gene $\operatorname{Yr} 29$ on chromosome $1 \mathrm{~B}$. This gene was closely linked with slow leaf rusting gene $L r 46$. Suenaga et al. (33) reported resistance gene $\operatorname{Yr} 30$ closely linked with durable adult plant stem rust resistance gene $S r 2$.

From the early 1970 s, the $1 B / 1 R$ translocation with $\operatorname{Yr} 9$ was introduced to Chinese wheat breeding programs through introductions such as Lovrin 10, Lovrin 13, Predgornaia 2, Kavkaz, and Neuzucht $(9,22)$. The widespread growing of cultivars with $\operatorname{Yr} 9$ resulted in major epidemics in 1990 and 2002, since the stripe rust resistance was overcome by the new race of the pathogen CYR 32, which has forced Chinese wheat breeders to focus on the development of cultivars with durable resistance. Although breeders are using slow rusting resistance and major genes for durable control of stripe rust, very little information is available on sources and efficacy of resistance in Chinese wheat cultivars.

A total of 145 widely grown Chinese wheat cultivars, newly released cultivars, and advanced lines from 12 provinces in the major wheat production regions were investigated in this study. The objectives were to identify probable genes conferring resistance to stripe rust at the seedling stage and to investigate the likelihood of slow rusting resistance from adult plant tests in the field.

\section{MATERIALS AND METHODS}

Wheat germ plasm. The wheat lines comprised 145 Chinese leading cultivars, newly released cultivars, and advanced lines from major breeding programs across the autumn-sown wheat regions of China, including the provinces of Hebei, Henan, Shandong, Shaanxi, Gansu, Anhui, Hubei, Jiangsu, Chongqing, Guizhou, Sichuan, and Yunnan. Ninety-eight lines were tested for seedling response to $26 P$. striiformis $\mathrm{f}$. sp. tritici pathotypes in the greenhouse, and 135 were tested for slow stripe rusting resistance in the field (Table 1).

Seedling testing. A set of 25 differential lines with known stripe rust resistance genes (20) were included in the seedling tests, and the infection types displayed by them were used as a basis for postulating resistance genes in the Chinese lines. The seedling tests for the 98 cultivars and lines were conducted in 2004 and 2005, respectively, using 26 pathotypes with the method described by Bariana and McIntosh (1) and Niu et al. (21) (Table 2). Six to eight plants of each line tested were grown in a growth chamber. Seedling inoculations were performed by brushing urediniospores of pathotypes from a fully infected susceptible cultivar onto the test seedlings when the first leaf was fully expanded. Inoculated seedlings were subsequently placed in plastic-covered cages and incubated at $9^{\circ} \mathrm{C}$ and $100 \% \mathrm{RH}$ for 24 h. Seedlings were then transferred to growth chambers with the day/night regime of $14 \mathrm{~h}$ of light $(22,000$ lux $)$ at $17^{\circ} \mathrm{C}$ and $10 \mathrm{~h}$ of darkness at $12^{\circ} \mathrm{C}$, with $70 \%$ RH. Infection types (ITs) were scored 15 to 16 days after inoculation based on a 0 to 4 scale (1) when rust was fully developed on the susceptible check, Mingxian 169. Plants with IT 0 to $2+$ were considered to be resistant, and those with IT of 3- to 4 , susceptible.

Identification of $1 \mathrm{~B} / 1 \mathrm{R}$ status. $1 \mathrm{~B} / 1 \mathrm{R}$ translocation lines were identified according to the method described by Fransis et al. (8).

Field testing. Of the 145 cultivars and lines employed in this study, 135 lines and slow rusting check Lantian 12 and highly susceptible check Tiaogan 601 were planted in a randomized complete block design with three replicates, in Beijing and Tianshui in the 2003-2004 and 2004-2005 cropping seasons for the test of adult-plant resistance (Table 1). Each plot consisted of a $2-\mathrm{m}$ row spaced $25 \mathrm{~cm}$ apart with 150 seeds. Beijing is the location of the main winter wheat breeding program. Tianshui, located in Gansu province, is considered a hot spot for stripe rust $(42,47)$. Cultivar Lantian 12, with typical slow rusting (47), and highly susceptible line Tiaogan 601 were used as slow rusting and susceptible checks, respectively. Spreader rows of Tiaogan 601 were planted perpendicular and adjacent to the rows of tested cultivars. Stripe rust epidemics were initiated by spraying aqueous suspensions of urediospores of $P$. striiformis $\mathrm{f}$. sp. tritici pathotype CYR32, to which a few drops of Tween $20(0.03 \%)$ had been added, onto the spreader rows at tillering. The stripe rust severity was assessed for the first time 4 weeks after inoculation and then at weekly intervals with four times in total using the modified Cobb scale (25), and infection types were scored using a 0 to 4 scale (1). The disease severity data were used to calculate the area under the disease progress curve (AUDPC) based on the method described by Broers et al. (3). AUDPC was calculated as:

$$
\text { AUDPC }=\sum_{i=1}^{n}\left[\left(X_{i}+X_{i+1}\right) / 2\right]\left(T_{i+1}-T_{i}\right)
$$

where $T_{i}=$ number of days after inoculation and $X_{i}=$ disease severity. Relative AUDPC (rAUDPC), calculated using the actual AUDPC divided by the AUDPC of susceptible check Tiaogan 601 (15,17), and final disease severity (FDS), when the susceptible check (Tiaogan 601) displayed maximum disease severity, were used for statistical analyses. The field data obtained in Beijing during the 2004-2005 cropping season was excluded from analysis due to inadequate infection.

Statistical analysis. The Statistical Analysis System (SAS) procedural PROC GLM was used to conduct analysis of variance with lines, environments that were the combination of location and year, and their interaction effects as fixed, and replicates nested in environments as random. Fisher's $F$ protected least significant difference (LSD) was used to separate the means for AUDPC and FDS among the lines tested. Lines that were seedling susceptible to CYR32 and showed lower or not significantly higher values of FDS and AUDPC than those of the slow rusting check in field trials were considered to be slow rusting lines of potential use for crop protection and breeding.

\section{RESULTS}

Seedling reaction. The reactions of the standard differential lines with known resistance genes to the 26 pathotypes are included in Table 2. Line $\mathrm{Yr} 15 / 6^{*}$ Avocet S displayed high resistance to all pathotypes tested. Lines $Y r 24 / 3^{*}$ Avocet S and Yr26/3* Avocet $\mathrm{S}$ showed a moderately susceptible reaction only to pathotype P4 (75078). Moro with $\mathrm{Yr} 10$ gave high infection types to pathotypes P4 (75078) and P10 (72107). Thus, Yr10, Yr15, Yr24, and Yr26 confer effective resistance against prevailing Chinese pathotypes (P22 to 26), whereas lines with $Y r 1$ and $Y r 6$ were susceptible to them.

The resistance genes $\mathrm{Yr} 2, \mathrm{Yr} 3 a, \mathrm{Yr} 4 a$, $Y r 6, Y r 7, Y r 9, Y r 26, Y r 27$, and $Y r S D$, either independently or in certain combinations, were postulated in 72 lines, whereas 26 accessions did not contain any known stripe rust resistance genes.

Eighteen lines possessed only $\operatorname{Yr}$, whereas another 24 lines possessed $\mathrm{Yr} 9$ plus one or more unknown resistance genes according to their reaction patterns (Table 2). All lines postulated to have $\operatorname{Yr} 9$ also amplified a SCAR marker for rye chromatin (8) (Tables 1 and 2).

Fifteen lines were postulated to have Yr26 on the basis that only P4 (75078) was virulent. Pedigree information (Table 1) indicated that four additional lines (L107 = LB 0438, L115 = Mian 2000-8, L125 = Nei 2938, and L134 = Xike 01015), highly resistant to all pathotypes, probably carry Yr26 and one more unknown gene.

Four lines $(\mathrm{L} 133=\mathrm{W} 7, \mathrm{~L} 41=$ Shaan 512, L52 = Xinong 914, and L106 = LB 0288) carried $\operatorname{Yr} 6$ based on their responses to the pathotypes avirulent to $\mathrm{Yr} 6 / 6^{*}$ Avocet S (Table 2). L133 conferred resistance to pathotypes lacking pathogenicity for $Y r 2$, indicating that it contained $Y r 2$ and Yr6. Two lines $(\mathrm{L} 82=351-15, \mathrm{~L} 130=$ R88) displayed low infection types to pathotypes avirulent to differentials Vilmorin 23 and Nord Desprez, indicating they may contain $\mathrm{Yr} 3 a$ and $\mathrm{Yr} 4 a$. In addition, line L82 also carried $Y r 2$, being resistant to pathotypes lacking virulence for Yr2. Three lines $($ L64 $=$ Annong 98005, L72 = An 96-8, and L109 = Lemai 3) carried $Y r S D$, displaying low ITs to the pathotypes avirulent to $Y r S D$. L92 (CD 1485-6) conferred resistance to all pathotypes avirulent to $Y r 7$ and some pathotypes virulent for $Y r 7$, indicating it carried $Y r 7$ and unknown resistance genes. 
Table 1. Pedigrees of 145 Chinese wheat cultivars and lines tested for stripe rust response and arranged according to province of origin

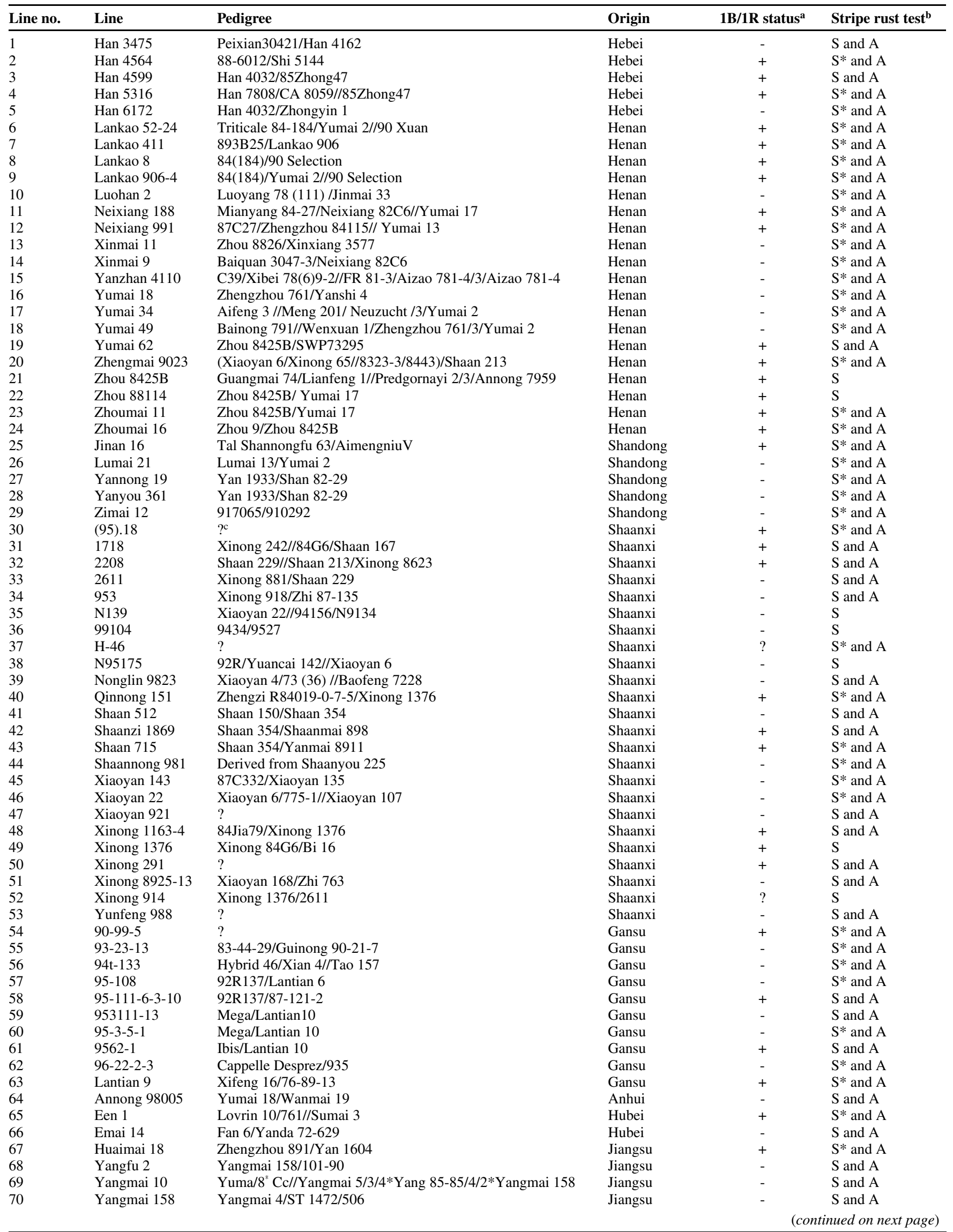

${ }^{\mathrm{a}}+=$ Presence of 1B/1R translocation, - = nonpresence of 1B/1R translocation, identified according to the method described by Fransis et al. (8).

${ }^{\text {b }}$ S, seedling tests with 26 PST pathotypes including CYR32, S*, seeding tests only with the pathotype CYR32, A, adult-plant tests.

c Unknown. 
Table 1. (continued from previous page)

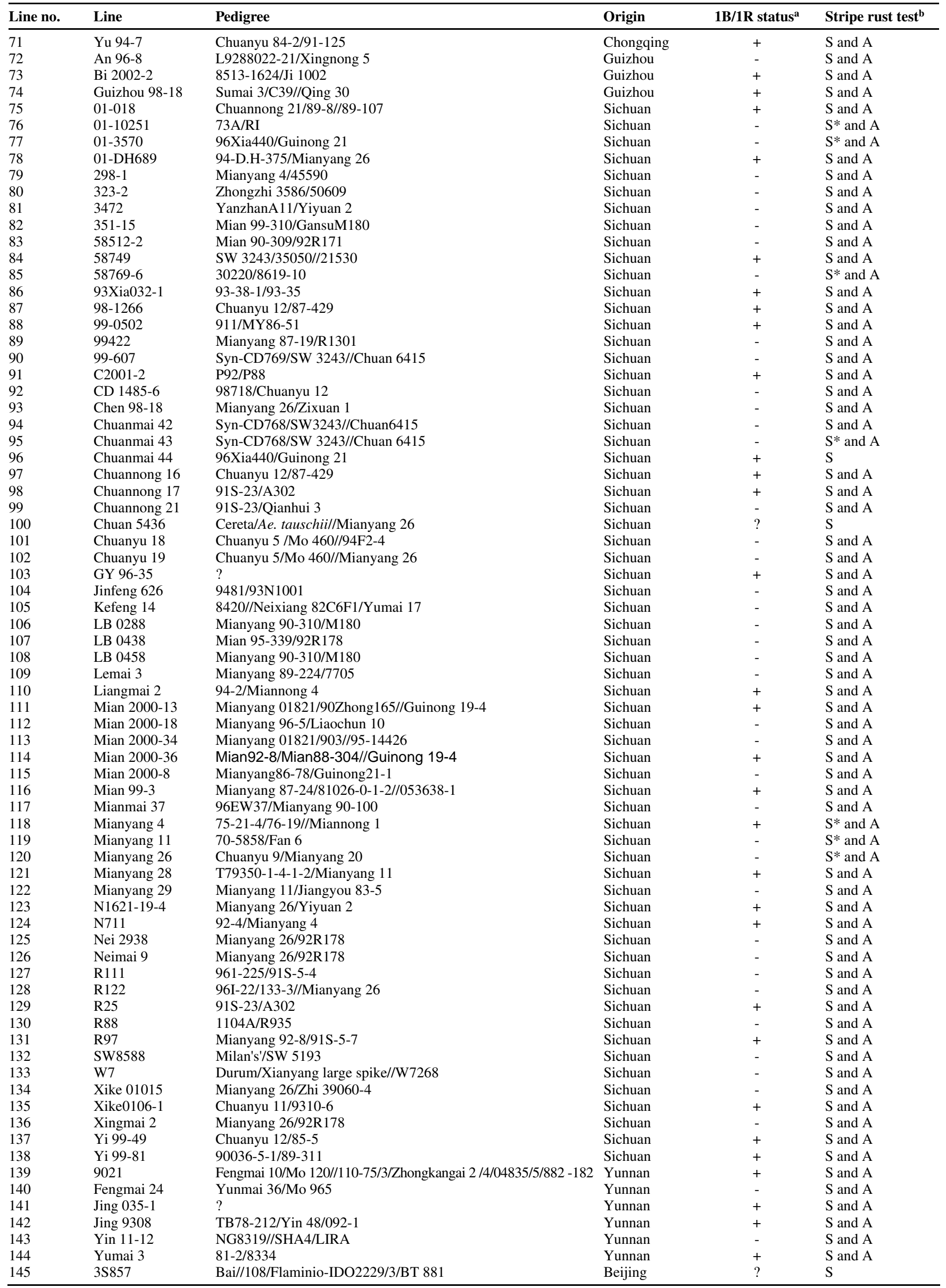


Table 2. Seedling infection types produced by 25 differentials (bold) with known resistance genes and 98 wheat cultivars and lines when infected with 26 pathotypes of Puccinia striiformis f. sp tritici collected from China and other countries ${ }^{\mathrm{a}}$

\begin{tabular}{|c|c|c|c|c|c|c|c|c|c|c|c|c|}
\hline \multirow[b]{2}{*}{ Line number } & \multirow[b]{2}{*}{ Yr gene } & \multicolumn{11}{|c|}{ Infection types to Puccinia striiformis f. sp. tritici pathotypes } \\
\hline & & $\begin{array}{c}61009 \\
(P 1)\end{array}$ & $\begin{array}{c}78028 \\
\text { (P2) }\end{array}$ & $\begin{array}{c}\mathbf{5 8 8 9 3} \\
(\mathrm{P3})\end{array}$ & $\begin{array}{c}75078 \\
\text { (P4) }\end{array}$ & $\begin{array}{c}60105 \\
\text { (P5) }\end{array}$ & $\begin{array}{c}\mathbf{5 9 7 9 1} \\
\text { (P6) }\end{array}$ & $\begin{array}{c}\mathbf{8 2 5 1 7} \\
\text { (P7) }\end{array}$ & $\begin{array}{c}78080 \\
(\mathrm{P8})\end{array}$ & $\begin{array}{c}\mathbf{8 6 0 9 4} \\
\text { (P9) }\end{array}$ & $\begin{array}{l}72107 \\
\text { (P10) }\end{array}$ & $\begin{array}{l}74187 \\
\text { (P11) }\end{array}$ \\
\hline Clement & Yr9, YrCle & $\mathbf{0}$ & $\mathbf{0}$ & $\mathbf{0}$ & $\mathbf{0}$ & $\mathbf{0}$ & $\mathbf{0}$ & $\mathbf{0}$ & 0 & 3 & 0 & 0 \\
\hline 19 & $Y r 9$ & 0 & 0 & 0 & 0 & 0 & 0 & 0 & 0 & 4 & 0 & 0 \\
\hline 42 & $Y r 9$ & 0 & 0 & 0 & 0 & 0 & 0 & 0 & 0 & 2 & 0 & 0 \\
\hline 71 & $Y r 9$ & 0 & 0 & 0 & 0 & 0 & 0 & 0 & 0 & 4 & 0 & 0 \\
\hline 78 & $Y r 9$ & 0,0 & 0 & 0 & 0 & 0 & 0 & 0,0 & 0 & 3 & 0 & 0 \\
\hline 84 & $\operatorname{Yr} 9$ & 0 & 0 & 0 & 0 & 0 & 0 & 0 & 0 & 3,4 & 0 & 0 \\
\hline 91 & $\operatorname{Yr} 9$ & 0 & 0 & 0,0 & 0 & 0 & 0 & 0 & 0 & 3 & 0 & 0 \\
\hline 110 & $\operatorname{Yr} 9$ & 0 & 0 & 0 & 0 & 0 & 0 & 0 & 0 & 4 & 0 & 0 \\
\hline 116 & $Y r 9$ & 0 & 0 & 0 & 0 & 0 & 0 & 0 & 0 & 4 & 0 & 0 \\
\hline 121 & $Y r 9$ & 0 & 0 & 0 & 0 & 0 & 0 & 0 & 0 & 3 & 0 & 0 \\
\hline 123 & $\operatorname{Yr} 9$ & 0 & 0 & 0 & 0 & 0 & 0 & 0 & 0 & 3 & 0 & 0 \\
\hline 135 & $\mathrm{Yr} 9$ & 0,0 & - & 0 & 0 & 0 & 0 & 0 & 0 & 4 & 0 & 0 \\
\hline 137 & $Y r 9$ & 0 & 0 & 0 & 0 & 0 & 0 & 0 & 0 & 4 & 0 & 0 \\
\hline 138 & $Y r 9$ & 0 & 0 & 0 & 0 & 0 & 0 & 0 & 0 & 4 & 0 & 0 \\
\hline 139 & $Y r 9$ & 0 & 0 & 0 & 0 & 0 & 0 & 0 & 0 & 3 & 0 & 0 \\
\hline 141 & $Y r 9$ & 0 & 0 & 0,0 & 0 & 0 & 0 & 0 & 0 & 3 & 0 & 0 \\
\hline 142 & $\mathrm{Yr} 9$ & 0 & 0 & 0 & 0 & 0,0 & 0 & 0 & 0 & 4 & 0 & 0 \\
\hline 143 & $Y r 9$ & 0 & 0 & 0 & 0 & 0 & 0 & 0 & 0 & 3 & 0 & 0 \\
\hline 144 & $Y r 9$ & 0,0 & 0 & 0 & 0 & 0 & 0 & 0 & 0 & 4 & 0 & 0 \\
\hline 3 & $\operatorname{Yr} 9,+$ & 0 & 0 & 0 & 0 & 0 & 0 & 0 & 0 & 0 & 0 & 0 \\
\hline 48 & $\operatorname{Yr} 9,+$ & 0 & 0 & 0 & 0 & 0 & 0 & 0 & 0 & 0 & 0 & 0 \\
\hline 50 & $\operatorname{Yr} 9,+$ & 0 & 0 & 0 & 0 & 0 & 0 & 0 & 0 & 0 & 0 & 0 \\
\hline 73 & $\operatorname{Yr} 9,+$ & 0,0 & 0 & 0 & 0 & 0 & 0,0 & 0 & 0 & 0 & 0 & 0 \\
\hline 75 & $\operatorname{Yr} 9,+$ & 0 & 0 & 0 & 0,0 & 0 & 0 & 0 & 0 & 0 & 0 & 0 \\
\hline 97 & $\operatorname{Yr} 9,+$ & 0 & 0 & 0 & 0 & 0 & 0,0 & 0 & 0 & 0,0 & 0 & 0 \\
\hline 98 & $\operatorname{Yr} 9,+$ & 0 & 0 & 0 & 0 & 0 & 0 & 0 & 0 & 0,0 & 0 & 0 \\
\hline 131 & $\operatorname{Yr} 9,+$ & 0 & 0 & 0,0 & 0 & 0 & 0 & 0 & 0 & 0,0 & 0 & 0 \\
\hline 129 & $\operatorname{Yr} 9,+$ & 0 & 0 & 0 & 0 & 0 & 0 & 0,0 & 0 & 0,0 & 0 & 0 \\
\hline 74 & $\operatorname{Yr} 9,+$ & 0 & 0 & 0 & 0 & 0 & 0 & 0 & 0 & 0 & 0 & 0,0 \\
\hline 88 & $\operatorname{Yr} 9,+$ & 0,0 & 0 & 0 & 0 & 0 & 0 & 0 & 0 & 0 & 0 & 0 \\
\hline 111 & $\operatorname{Yr} 9,+$ & 0 & 0,0 & 0 & 0 & 0 & 0 & 0 & 0 & 0 & 0 & 0,0 \\
\hline 124 & $\operatorname{Yr} 9,+$ & 0 & 0,0 & 0 & 0 & 0 & 0 & 0 & 0 & 0 & 0 & 0 \\
\hline 61 & $Y r 9,+$ & 0 & 0 & 0 & 0 & 0 & 0 & 0 & 0 & $0 ;, 1$ & 0 & 0 \\
\hline 87 & $\operatorname{Yr} 9,+$ & 0 & 0 & 0 & 0 & 0 & 0 & 0 & 0 & $0 ;, 1$ & 0 & 0 \\
\hline 21 & $\operatorname{Yr} 9,+$ & 0 & 0,0 & 0 & 0 & 0 & 0 & 0 & 0 & 2 & 0 & 0 \\
\hline 49 & $\operatorname{Yr} 9,+$ & 0 & 0 & 0 & 0 & 0 & 0,0 & 0 & 0 & 2 & 0 & 0 \\
\hline 22 & $\operatorname{Yr} 9,+$ & 0 & 0,0 & 0 & 0 & 0 & 0 & 0 & 0 & 3 & 0 & 0 \\
\hline 31 & $\operatorname{Yr} 9,+$ & 0 & 0 & 0 & 0 & 0 & 0 & 0 & 0 & 3 & 0 & 0 \\
\hline 114 & $\operatorname{Yr} 9,+$ & 0 & 0 & 0 & 0 & 0 & 0 & 0 & 0 & 3 & 0 & 0 \\
\hline 32 & $Y r 9,+$ & 0 & 0 & 0 & 0 & 0 & 0 & 0 & 0 & 4 & 0 & 0 \\
\hline 59 & $\operatorname{Yr} 9,+$ & 0 & 0 & 0 & 0 & 0 & 0 & 0 & 0 & 4 & 0 & 0,0 \\
\hline 86 & $\operatorname{Yr} 9,+$ & 0 & 0 & 0 & 0 & 0 & 0 & 0 & 0 & 4 & 0 & 0 \\
\hline 103 & $\operatorname{Yr} 9,+$ & 0,0 & 0 & 0 & 0 & 0 & 0 & 0 & 0 & 4 & 0 & 0 \\
\hline Yr24/3*Avocet & Yr24 & $\mathbf{0}$ & 0; & $\mathbf{0}$; & $2+$ & $\mathbf{0}$; & $0 ;, 1$ & 0; & $\mathbf{0}$; & $0 ;, 1$ & $0 ;, 1+$ & $\mathbf{0}$ \\
\hline Yr26/3*Avocet & Yr26 & $\mathbf{0}$ & 0; & 0; & $2+, 3$ & 0; & $0 ;, 1$ & 0; & 0; & $\mathbf{0}$ & $0 ;, 1$ & $\mathbf{0}$ \\
\hline 36 & $\operatorname{Yr} 26$ & 0 & 0 & 0 & $2+, 3$ & 0,0 & 0,0 & $0 ;, 1+$ & 0 & 0 & 2 & 0 \\
\hline 38 & $\operatorname{Yr} 26$ & 0,0 & 0 & 0 & 3 & 0 & 0,0 & 0 & 0 & 0,0 & $2+$ & 0 \\
\hline 58 & $\operatorname{Yr} 26$ & 0 & 0 & 0 & 3 & 0 & 0 & 0 & 0 & 0 & 0 & 0 \\
\hline 83 & $\operatorname{Yr} 26$ & 0 & 0 & 0 & 3,4 & 0 & 0 & 0 & 0 & 0 & $0 ;, 1$ & 0 \\
\hline 90 & Yr26 & 0 & 0,0 & 0 & $2+, 3$ & 0,0 & 0 & 0 & 0 & 0 & 2 & 0 \\
\hline 94 & Yr26 & 0,0 & 0,0 & 0 & $2+, 3$ & 0 & 0 & 0 & 0 & 0 & $0 ;, 1$ & 0 \\
\hline 96 & Yr26 & 0 & 0 & 0 & 2 & 0 & 0 & 0 & 0 & 0 & $0 ;, 1$ & 0 \\
\hline 100 & Yr26 & 0,0 & 0,0 & 0 & $2+, 3$ & 0 & 0 & 0 & 0 & 0 & $0 ;, 1$ & 0 \\
\hline 104 & $\operatorname{Yr} 26$ & 0 & 0 & 0 & 3 & 0 & 0 & 0,0 & 0 & 0 & 0 & 0,0 \\
\hline 113 & Yr26 & 0 & 0 & 0,0 & 2 & 0 & 0 & 0 & 0 & 0 & 0 & 0 \\
\hline 117 & $\operatorname{Yr} 26$ & 0 & 0 & 0 & 3 & 0 & 0 & 0,0 & 0 & 0,0 & 0 & 0 \\
\hline 126 & Yr26 & 0 & 0 & 0 & 2,3 & 0 & 0 & 0 & 0 & 0 & 0 & 0 \\
\hline 127 & $\operatorname{Yr} 26$ & 0,0 & 0 & 0 & 3 & 0,0 & 0 & 0 & 0 & 0,0 & $0 ;, 1$ & 0 \\
\hline 128 & $\operatorname{Yr} 26$ & 0,0 & 0,0 & 0 & 3 & 0 & 0 & 0 & 0 & 0,0 & $0 ;, 1$ & 0 \\
\hline 136 & $\operatorname{Yr} 26$ & 0,0 & 0 & 0 & 3 & 0 & 0 & 0 & 0 & 0 & 1 & 0,0 \\
\hline 107 & $\operatorname{Yr} 26,+$ & 0 & 0 & 0 & 0 & 0 & 0 & 0 & $0 ;, 1$ & 0 & 0 & 0 \\
\hline 115 & $\operatorname{Yr} 26,+$ & 0 & 0,0 & 0 & 0 & 0 & 0 & 0 & 0 & 0,0 & 0 & 0,0 \\
\hline 125 & $\operatorname{Yr} 26,+$ & 0 & 0 & 0 & $0+$ & 0 & 0 & 0 & 0 & 0 & 0 & 0 \\
\hline 134 & $\operatorname{Yr26},+$ & 0,0 & 0 & 0 & $0 ;, 1,2$ & 0 & 0 & 0 & 0 & 0 & $1,1+$ & 0,0 \\
\hline $\begin{array}{l}\text { Maris } \\
\text { Huntsman }\end{array}$ & $\underset{Y r 2, \underset{Y r}{Y} 3 a, Y r 4 a}{\text { Yris }}$ & $\mathbf{0}$ & 0; & $0 ;, 1+$ & 3 & $0 ;, 1+$ & 3 & 4 & 4 & $\mathbf{0}$ & $\mathbf{0}$ & 3 \\
\hline 82 & Yr2, Yr3a, Yr4a,+ & 0,0 & 0 & 0 & 0 & 0 & 0 & 0 & $0 ;, 2$ & 0,0 & 1 & 0 \\
\hline Heines VII & Yr 2 & $0 ;, 1$ & $1,2+$ & $0 ;, 1+$ & $3,3+$ & $0 ;, 2+$ & 4 & 4 & 3 & 0 & $0 ;, 1$ & $\mathbf{0}$ \\
\hline
\end{tabular}

a Lines are arranged in groups according to postulated $\operatorname{Yr}$ genes. $+=$ unknown gene(s); - = untested. 


\begin{tabular}{|c|c|c|c|c|c|c|c|c|c|c|c|c|c|c|}
\hline \multicolumn{15}{|c|}{ Infection types to Puccinia striiformis f. sp. tritici pathotypes } \\
\hline $\begin{array}{l}86036 \\
(\mathrm{P} 12)\end{array}$ & $\begin{array}{l}76088 \\
(\mathrm{P} 13)\end{array}$ & $\begin{array}{l}76093 \\
(\mathbf{P} 14)\end{array}$ & $\begin{array}{c}86106 \\
(\mathbf{P} 15)\end{array}$ & $\begin{array}{l}68009 \\
(\mathrm{P} 16)\end{array}$ & $\begin{array}{r}86107 \\
(\mathbf{P 1 7})\end{array}$ & $\begin{array}{l}82061 \\
(\text { P18) }\end{array}$ & $\begin{array}{l}80551 \\
(\mathbf{P 1 9})\end{array}$ & $\begin{array}{r}85019 \\
(\mathbf{P 2 0})\end{array}$ & $\begin{array}{l}\text { PE92 } \\
\text { (P21) }\end{array}$ & $\underset{(\text { P22) }}{\text { CYR26 }}$ & $\begin{array}{c}\text { CYR27 } \\
\text { (P23) }\end{array}$ & $\begin{array}{c}\text { CYR29 } \\
\text { (P24) }\end{array}$ & $\begin{array}{l}\text { CYRSu- } \\
1 \text { (P25) }\end{array}$ & $\begin{array}{c}\text { CYR32 } \\
\text { (P26) }\end{array}$ \\
\hline $\mathbf{0}$ & $\mathbf{0}$ & $\mathbf{0}$ & $\mathbf{0}$ & $\mathbf{0}$ & 4 & 4 & 4 & 0; & $\mathbf{0}$ & $\mathbf{0}$ & $\mathbf{0}$ & 4 & 0; & 4 \\
\hline 0 & 0,0 & 0 & 0 & 0 & 4 & 4 & 4 & 0 & 0 & 0 & 0 & 4 & 0 & 4 \\
\hline 0 & 0 & 0 & 0 & 0 & 3 & 2 & 3 & 0 & 0 & 0 & 0 & 4 & 0 & 4 \\
\hline 0 & 0 & 0,0 & 0 & 0 & 4 & 3 & 4 & 0 & 0 & 0 & 0 & 4 & 0 & 4 \\
\hline 0,0 & 0 & 0 & 0 & 0 & 3 & 4 & 4 & 0,0 & 0 & 0 & 0 & $2,2+$ & 0 & 3 \\
\hline 0 & 0 & 0 & 0 & 0 & 4 & 3 & 3 & 0 & 0 & 0 & 0 & 4 & 0 & 4 \\
\hline 0,0 & 0 & 0 & 0 & 0 & 3 & 3 & 3 & 0 & 0 & 0,0 & 0 & 3 & 0 & 4 \\
\hline 0 & 0 & 0 & 0 & 0 & 3 & 3 & 4 & 0 & 0 & 0,0 & 0 & 4 & 0 & 3 \\
\hline 0 & 0 & 0,0 & 0 & 0 & 4 & 4 & 4 & 0 & 0 & 0 & 0 & 4 & 0 & 4 \\
\hline 0,0 & 0 & 0,0 & 0 & 0 & 4 & 3 & 4 & 0 & 0 & 0 & 0 & 4 & 0 & 4 \\
\hline 0 & 0 & 0 & 0 & 0 & 3 & 4 & 2 & 0,0 & 0 & 0 & 0 & 3 & 0 & 3 \\
\hline 0 & 0 & 0 & 0 & 0 & 4 & 4 & 4 & 0,0 & 0 & 0 & 0 & 4 & 0 & 4 \\
\hline 0 & 0 & 0 & 0 & 0 & 3 & 4 & 4 & 0 & 0 & 0 & 0 & 4 & 0 & 4 \\
\hline 0 & 0 & 0 & 0 & 0 & 3 & 3 & 3 & 0 & 0 & 0 & 0 & $3+$ & 0 & 4 \\
\hline 0 & 0 & 0 & 0 & 0 & 3 & 4 & 4 & 0 & 0 & 0,0 & 0 & 4 & 0 & 3 \\
\hline 0 & 0,0 & 0,0 & 0 & 0 & 3 & 4 & 4 & 0,0 & 0 & 0 & 0 & 3 & 0 & 4 \\
\hline 0 & 0 & 0 & 0,0 & 0 & 3 & 3 & 4 & 0,0 & 0 & 0 & 0 & 4 & 0 & 4 \\
\hline 0 & 0,0 & 0 & 0 & 0 & 3 & 3 & 4 & 0 & 0 & 0,0 & 0 & 4 & 0 & 4 \\
\hline 0 & 0 & 0 & 0 & 0 & 4 & 4 & 4 & 0 & 0 & 0,0 & 0 & 4 & 0 & 3 \\
\hline 0 & 0 & 0 & 0 & 0 & 3 & 0 & 3 & 0 & 0 & 0,0 & 0 & 4 & 0,0 & 4 \\
\hline 0 & 0 & 0 & 0 & 0 & $0 ;, 1$ & 3 & 3 & 0 & 0 & 0 & 0 & 0 & 0 & 4 \\
\hline 0,0 & 0 & 0 & 0 & 0 & 3 & 0 & $0 ;, 1$ & 0 & 0 & 0 & 0 & 4 & 0 & $3+$ \\
\hline 0 & 0,0 & 0 & 0 & 0 & 1,2 & 0 & $0 ;, 2$ & 0 & 0 & 0 & 0 & $3+$ & 0 & 3 \\
\hline 0 & 0 & 0 & 0 & 0 & 0 & 3 & 0,0 & 0 & 0 & 0 & 0 & 0 & 0 & 3 \\
\hline 0 & 0 & 0 & 0 & 0 & $0 ;, 1$ & 3 & 0 & 0 & 0,0 & 0 & 0 & $1+, 2+$ & 0 & 3,4 \\
\hline 0 & 0 & 0 & 0 & 0 & $0 ;, 1$ & 3 & 0 & 0 & 0 & 0 & 0 & 0 & 0 & 3 \\
\hline 0 & 0 & 0 & 0 & 0 & 0 & 3 & 0 & 0 & $0,1+$ & 0 & 0 & 0 & 0 & 3 \\
\hline 0 & 0 & 0 & 0 & 0 & $0 ;, 1$ & 3 & 0,0 & 0 & 0 & 0 & 0 & 0 & 0 & $2,2+$ \\
\hline 0 & 0 & 0 & 0 & 0 & 3 & 2 & $3+$ & 0 & 0 & 0,0 & 0 & 4 & 0 & 3 \\
\hline 0 & 0 & 0 & 0 & 0 & 0 & 3 & 0 & 0 & 0 & 0 & 0 & 0 & 0 & 4 \\
\hline 0 & 0 & 0 & 0 & 0 & 1,2 & $0 ;, 2$ & $0 ;, 1$ & 0 & 0 & 0 & 0 & 0 & 0 & 4 \\
\hline 0 & 0 & 0 & 0 & 0 & 0 & 0 & $0 ;, 1$ & 0 & 0 & 0 & 0 & 4 & 0 & 4 \\
\hline 0 & 0 & 0 & 0 & 0 & 2 & 0 & $3,3+$ & 0 & 0,0 & 0 & 0 & 0 & 0 & 2 \\
\hline 0 & 0 & 0 & 0 & 0 & 1 & 3 & 0,0 & 0 & 0 & 0 & 0 & $0 ;, 2+$ & 0 & $2+$ \\
\hline 0 & 0 & 0 & 0 & 0 & 2 & 3 & $3+, 4$ & 0 & 0 & 0 & 0 & $1+, 2$ & 0 & $1+, 2$ \\
\hline 0 & 0 & 0 & 0 & 0 & $2+$ & 0,0 & 0 & 0,0 & 0 & 0,0 & 0 & 4 & 0 & 4 \\
\hline 0 & 0 & 0 & 0 & 0 & 2 & 3 & $3+, 4$ & 0 & 0 & 0 & 0 & $1+, 2$ & 0 & $1+, 2$ \\
\hline 0 & 0 & 0 & 0 & 0 & 4 & 0,0 & 0 & 0 & 0 & 0 & 0 & 4 & 0 & 4 \\
\hline 0 & 0 & 0,0 & 0 & 0 & 4 & $0 ;, 2$ & $0 ;, 1$ & 0 & 0 & 0 & 0 & 4 & 0 & 4 \\
\hline 0 & 0 & 0 & 0 & 0 & 4 & 0 & 4 & 0 & 0 & 0 & 0 & 3 & 0 & 4 \\
\hline 0 & 0 & 0 & 0 & 0 & 4 & 0 & 1,2 & 0 & 0 & 0 & 0 & 4 & 0 & 4 \\
\hline 0 & 0 & 0 & 0,0 & 0 & 0 & 3 & 4 & 0 & 0 & 0 & 0 & $2,2+$ & 0 & 3 \\
\hline 0 & 0,0 & 0 & 0 & 0 & 3 & 0 & 4 & 0 & 0 & 0 & 0 & 3 & 0 & 3,4 \\
\hline 0; & o & $0 ;, 1+$ & 0; & 0; & 0; & 0; & 0; & $0 ;, 1$ & 0; & 0; & 0; & 0; & 0; & $\mathbf{0}$ \\
\hline 0; & 0; & 0; & $0 ;, 2$ & 0; & 0; & 0; & 0; & $0 ;, 1$ & 0; & 0; & 0; & 0; & 0; & 0; \\
\hline 0 & 0 & 0,0 & 0 & 0 & 0,0 & 0 & 0 & 0 & 0 & 0 & 0 & 0 & 0 & 0 \\
\hline 0 & 0 & 0 & 0 & 0 & 0 & 0 & 0 & 0 & 0 & 0 & 0 & 0 & 0,0 & 0 \\
\hline 0 & 0,0 & 0,0 & 0 & 0 & 0 & 0 & 0 & 0 & 0 & 0 & 0 & 0 & 0 & 0,0 \\
\hline 0 & 0,0 & 0 & $0 ;, 1+$ & 0 & 0 & 0 & 0 & $0 ;, 1$ & 0 & 0,0 & 0 & 0,0 & 0 & 0 \\
\hline 0 & 0 & 0 & 0 & 0 & 0 & 0 & 0 & 0 & 0 & 0 & 0 & 0 & 0 & 0 \\
\hline 0 & 0 & 0 & 0 & 0 & 0 & 0 & 0 & 0 & 0 & 0 & 0,0 & 0 & 0 & 0 \\
\hline 0 & 0 & 0 & 0 & 0 & 0 & 0 & 0 & 0 & 0 & 0 & 0 & 0 & 0,0 & 0 \\
\hline 0 & 0 & 0 & 0 & 0 & 0 & 0 & 0 & 0 & 0 & 0 & 0 & 0 & 0 & 0 \\
\hline 0 & 0,0 & 0 & 0 & 0 & 0 & 0 & 0 & 0 & 0 & 0 & 0 & 0 & 0,0 & 0 \\
\hline 0 & 0 & 0 & 0,0 & $0 ;, 1$ & 0 & 0 & 0 & 0 & 0 & 0 & 0 & 0 & 0 & 0 \\
\hline 0 & 0 & 0,0 & 0 & 0 & 0 & 0 & 0 & 0 & 0 & 0 & 0 & 0,0 & 0 & 0,0 \\
\hline 0 & 0 & 0 & 0 & 0 & 0,0 & 0 & 0 & $0 ;, 1$ & 0 & 0 & 0 & 0 & 0 & 0 \\
\hline 0,0 & 0,0 & 0 & 0,0 & 0 & 0 & 0 & 0 & 0 & 0 & 0 & 0 & 0 & 0 & $0 ;, 1+$ \\
\hline 0 & 0 & 0,0 & $0 ;, 1+$ & 0 & 0 & 0 & 0 & 0 & 0 & 0 & 0 & 0 & 0 & 0 \\
\hline 0 & 0 & 0 & $0 ;, 2$ & 0 & 0,0 & 0 & 0 & 0 & 0 & 0 & 0 & 0 & 0 & 0 \\
\hline 0 & 0 & 0 & 0 & 0 & 0 & 0 & 0 & 0 & 0,0 & 0 & 0 & 0 & 0 & 0 \\
\hline 0 & 0,0 & 0 & $0 ;, 1$ & 0 & 0,0 & $0 ;, 1$ & 0 & 0 & 0 & 0 & 0 & 0 & 0,0 & 0 \\
\hline 0 & 0,0 & 0 & 0 & 0 & 0 & 0 & 0 & 0 & 0 & 0 & 0 & 0 & 0 & 0 \\
\hline 0,0 & 0,0 & 0,0 & 0 & 0 & 0 & 0 & 0 & 0 & 0 & 0 & 0 & 0 & 0 & 0 \\
\hline $\mathbf{0}$ & 0 & $\mathbf{0}$ & $0 ;, 1+$ & 4 & 0; & 4 & 3 & 4 & $1+, 2+$ & $3+$ & 3 & 4 & $\mathbf{0}$ & $3+, 4$ \\
\hline 0 & 0 & 0 & 0 & 0 & 0 & 2 & 0 & 3 & 0 & 3 & 3 & 0,1 & 0 & 4 \\
\hline \multirow[t]{2}{*}{ 0; } & 0; & $1+$ & 4 & 4 & 3 & 4 & 3 & 2 & 2 & 3 & 3 & 4 & 1 & 4 \\
\hline & & & & & & & & & & & & \multicolumn{3}{|c|}{ (continued on next page) } \\
\hline
\end{tabular}




\begin{tabular}{|c|c|c|c|c|c|c|c|c|c|c|c|c|}
\hline \multirow[b]{2}{*}{ Line number } & \multirow[b]{2}{*}{ Yr gene } & \multicolumn{11}{|c|}{ Infection types to Puccinia striiformis f. sp. tritici pathotypes } \\
\hline & & $\begin{array}{c}61009 \\
(P 1)\end{array}$ & $\begin{array}{c}78028 \\
\text { (P2) }\end{array}$ & $\begin{array}{c}58893 \\
\text { (P3) }\end{array}$ & $\begin{array}{c}75078 \\
(\mathbf{P 4})\end{array}$ & $\begin{array}{c}60105 \\
\text { (P5) }\end{array}$ & $\begin{array}{c}59791 \\
\text { (P6) }\end{array}$ & $\begin{array}{c}\mathbf{8 2 5 1 7} \\
\text { (P7) }\end{array}$ & $\begin{array}{c}78080 \\
(\mathrm{P8})\end{array}$ & $\begin{array}{c}86094 \\
\text { (P9) }\end{array}$ & $\begin{array}{l}72107 \\
\text { (P10) }\end{array}$ & $\begin{array}{l}74187 \\
\text { (P11) }\end{array}$ \\
\hline Yr6/6*Avocet S & Yr6 & 3 & 4 & $1,1+$ & 3 & 0; & 0; & $1+, 2$ & 4 & 4 & $2+$ & 4 \\
\hline 133 & $\operatorname{Yr} 2, \operatorname{Yr} 6,+$ & 0 & 0 & 0 & 0,0 & 0 & 0 & 0 & 2,0 & 0 & $1^{+}$ & $0 ;, 1$ \\
\hline 41 & $\operatorname{Yr} 6,+$ & 3 & 0 & $0 ;, 2$ & 4 & 0 & 0 & $0 ;, 1^{+}$ & 3 & 3 & 3 & 0 \\
\hline 52 & $\operatorname{Yr} 6,+$ & 0 & 2 & $0 ;, 1$ & 0 & 0 & 0 & 0 & 0 & 4 & 0 & 0 \\
\hline 106 & $\operatorname{Yr6},+$ & 0 & 3 & 0,0 & $0 ;, 1,2$ & 0,0 & 0 & 0 & $0 ;, 1$ & 0,0 & 1 & 0 \\
\hline Vilmorin 23 & Yr3a, Yr4a, & 4 & 0; & 0; & 3 & $0 ;, 2+$ & 1 & 4 & 4 & 1 & 0; & 3 \\
\hline Nord Desprez & Yr3a, Yr4a, & 4 & 1,2 & $0 ;, 1+$ & 3 & $0 ;, 1$ & $2^{+}$ & 4 & 4 & 2 & 0; & 3 \\
\hline 130 & $\operatorname{Yr} 3 a, \operatorname{Yr} 4 a,+$ & 2 & $0 ;, 1$ & $1,1+$ & $2^{+}$ & $1,2+$ & 0 & $3+$ & 0 & 2 & $0 ;, 1$ & 3 \\
\hline Lee* & Yr7, Yr22 & 0; & 4 & 0; & $2+, 3$ & $0 ;, 1$ & 0; & $0 ;, 1$ & 0; & 4 & 4 & 0; \\
\hline Reichersberg 42 & Yr7 & 0; & 4 & 0; & $1+$ & $0 ;, 1$ & 0; & $0 ;, 1$ & 0; & $2+$ & $\mathbf{0}$ & $0 ;, 1$ \\
\hline 92 & $Y r 7,+$ & 2 & $2+, 3$ & $0 ;, 1+$ & $3^{+}$ & 2 & 0 & 2 & 0 & 1,2 & $3+$ & $0 ;, 1$ \\
\hline Yr15/6*Avocet S & $\operatorname{Yr} 15$ & 0; & $\mathbf{0}$ & 0 & $\mathbf{0}$ & $\mathbf{0}$ & $\mathbf{0}$ & $\mathbf{0}$ & $\mathbf{0}$ & $\mathbf{0}$ & $\mathbf{0}$ & 0 \\
\hline 35 & Yr15 or others & 0 & 0 & 0 & 0 & 0,0 & 0 & 0 & 0 & 0 & 0 & 0 \\
\hline 80 & Yr15 or others & 0 & 0,0 & 0 & 0 & 0 & 0 & 0 & 0 & 0 & 0 & 0 \\
\hline 145 & Yr15 or others & 0 & 0 & 0 & 0 & 0 & 0 & 0 & 0 & 0 & 0 & 0 \\
\hline Selkirk & Yr27 & 1 & $2,2+$ & 3 & 3 & 3 & 0; & 2 & 2 & 2 & 4 & 3 \\
\hline 112 & $\operatorname{Yr} 27,+$ & 0 & 0,0 & 0 & 3 & 0,0 & 0 & 0 & 0 & 0,0 & 4 & 0 \\
\hline $\begin{array}{l}\text { Strubes } \\
\text { Dickkopf }\end{array}$ & YrSD & 4 & $0 ; 1+$ & 4 & 3 & 4 & 4 & 4 & 4 & 3 & $\mathbf{0} ; \mathbf{1}$ & $\mathbf{0}$ \\
\hline 64 & $\operatorname{YrSD},+$ & 3 & $0 ;, 1+$ & $1+, 2$ & 3 & 1,2 & 2 & 4 & 0 & 3 & 0 & 0 \\
\hline 72 & $\operatorname{YrSD},+$ & 4 & $0 ;, 1$ & $0 ;, 2$ & 3 & 1,2 & 0 & 3 & 3 & 4 & 0 & 0 \\
\hline 109 & $Y r S D,+$ & 3 & 0,0 & 0 & 0,2 & 1,2 & $0 ;, 1,2$ & 3 & 3 & 0,0 & $1,1+$ & 0,0 \\
\hline Chinese 166 & Yr1 & 0; & $\mathbf{0}, \mathbf{0}$ & 0; & $\mathbf{0}$ & $3^{+}$ & 0; & 4 & $\mathbf{0}$ & 0 & 3 & 0 \\
\hline Hybrid 46 & $Y r 3 b, Y r 4 b$, & 0; & 0; & $0 ;, 1$ & $3,3^{+}$ & 0; & 0; & $2+, 3$ & 4 & 0; & 0; & $0 ; 1$ \\
\hline Compair & Yr8 & 0; & 4 & $\mathbf{0}, \mathbf{0}$ & 4 & 0 & 0 & $\mathbf{0}, \mathbf{0}$ & $\mathbf{0}$ & 3 & 4 & $\mathbf{0}$ \\
\hline Moro & Yr10 & 0 & $\mathbf{0}$ & $\mathbf{0}, \mathbf{0}$ & $3^{+}, 4$ & $\mathbf{0}$ & $\mathbf{0}$ & 0 & $\mathbf{0}$ & $\mathbf{0}$ & 4 & 0 \\
\hline VPM & Yr17 & 0; & $2+, 3$ & 0; & 1,2 & 1,2 & $0 ;, 1$ & $1+, 2$ & 2 & 0; & 0 & 0 \\
\hline Funo & $Y r A$ & 3 & $3+, 4$ & 0; & $3,3+$ & $0 ;, 1$ & 3 & $2+$ & 3 & 4 & $1,1+$ & 3 \\
\hline Alba* & YrAlba & 3 & 0; & $0 ;, 1+$ & $3,3+$ & 3 & 3,4 & $3+$ & 4 & 2 & 0; & $0 ;, 1$ \\
\hline Carstens V & $\operatorname{YrCV}$ & 3 & 0 & $2^{+}$ & 3 & 4 & 0; & 3 & 3 & 4 & $2+$ & 4 \\
\hline Gaby & YrGaby & 0; & $2+$ & 0; & 0; & 0; & 0; & 0; & 4 & 3 & 0; & 0; \\
\hline Resulka & YrRes & 0 & $\mathbf{0}$ & $\mathbf{0}$ & 0; & 0; & $\mathbf{0}$ & 0 & $\mathbf{0}$ & $\mathbf{0}$ & 0 & $\mathbf{0}$ \\
\hline $\begin{array}{l}\text { Spaldings } \\
\text { Prolific }\end{array}$ & YrSpp & $\mathbf{0}$ & 0; & $\mathbf{0} ; \mathbf{1}$ & $2+, 3$ & 2,3 & $\mathbf{0}$ & 0; & 1 & 0 & 0 & 3 \\
\hline Suwon 92/Omar & YrSu & $\mathbf{0 , 0}$ & 0; & 0; & 4 & 0; & $\mathbf{0}$ & $0 ;, 1+$ & 4 & $\mathbf{0}$ & 4 & 4 \\
\hline 1 & + & 3 & 3 & 4 & 3 & 1,2 & 3 & 4 & 4 & 3 & 4 & 3 \\
\hline 33 & + & 3 & 3 & $3,3+$ & 3 & 3 & 0 & $3+$ & 0 & 0 & $2+$ & 3 \\
\hline 34 & + & 0 & 0 & $2,2+$ & 2 & 3 & 0,0 & 3 & 0 & 4 & 3 & 3 \\
\hline 39 & + & $0 ;, 2$ & 0 & 3 & 3 & $2^{+}$ & 0,0 & 3 & 3 & 3 & 3 & 3 \\
\hline 47 & + & 4 & 3 & 4 & 4 & 4 & 4 & 4 & 3 & 4 & 3 & 4 \\
\hline 51 & + & 3 & 0 & 3 & 4 & 3 & 2,3 & 4 & 3 & $2+$ & 3 & 1 \\
\hline 53 & + & 4 & 4 & 4 & 3 & 3 & 4 & 3 & 0 & 3 & 4 & 3 \\
\hline 66 & + & 3 & 3 & 4 & 3 & 4 & 3 & 3 & 3 & 3 & 3 & 3 \\
\hline 68 & + & 4 & 3 & 4 & 4 & 4 & 3 & 4 & 4 & 4 & 4 & 4 \\
\hline 69 & + & 4 & 3 & 4 & 4 & 4 & 4 & 4 & 3 & 4 & 4 & 4 \\
\hline 70 & + & 4 & 3 & 4 & 4 & 4 & 4 & 4 & 4 & 4 & 4 & 4 \\
\hline 79 & + & 4 & $0 ;, 1+$ & $3,3+$ & $3+$ & $3+, 4$ & 0 & 3 & 3 & 3 & $1+, 2$ & 4 \\
\hline 81 & + & 1,2 & 3 & 3 & 3 & $0 ;, 1$ & 0 & 3 & 3 & $0 ;, 2$ & 0 & 3 \\
\hline 89 & + & 4 & 4 & 4 & 4 & 4 & 4 & 3 & 3 & 3 & 4 & 4 \\
\hline 93 & + & $0 ;, 2$ & 3 & 3 & 2 & 1,2 & 0 & 3 & 3 & 2 & 0 & 2 \\
\hline 99 & + & 3,4 & 0 & 4 & 4 & 3 & 0 & 0,2 & 1,2 & 3 & 3 & 3 \\
\hline 101 & + & 4 & 4 & 3 & 4 & 3 & 1,2 & 3 & 3 & 4 & 3 & 3 \\
\hline 102 & + & 4 & 3 & 3 & 4 & 3 & 3 & 3 & 3 & 4 & 3 & 4 \\
\hline 105 & + & 4 & 4 & 4 & 4 & 4 & 4 & 4 & 4 & 4 & 4 & 4 \\
\hline 108 & + & 3 & 4 & 4 & $3+$ & 0 & 0 & $0 ;, 1$ & $0 ;, 1$ & 3 & 3 & 4 \\
\hline 122 & + & 4 & 3 & 3 & 4 & 3 & 3 & 4 & $3+$ & $2+$ & 4 & 4 \\
\hline 132 & + & 0,0 & 0 & 0,0 & 0,0 & 0 & 0 & 0 & 0 & 0,0 & 4 & 0 \\
\hline 140 & + & 3 & 0 & 4 & $3+$ & $0 ;, 2$ & 3 & 3 & 4 & 2 & 3 & $0 ;, 2$ \\
\hline
\end{tabular}

Low infection types produced by L112 (Mian 2000-18) to pathotypes avirulent to Selkirk (Yr27) and some pathotypes virulent to $Y r 27$ indicated the presence of $Y r 27$ and other unidentified resistance genes.

Six lines $(\mathrm{L} 47=$ Xiaoyan 921, L68 = Yangfu 2, L69 = Yangmai 10, L70 = Yangmai 158, L89 = 99422, and L102 = Chuanyu 19) showed high infection types to all pathotypes tested, indicating they did not have any detectable stripe rust resistance genes present in the reference differ- entials. The reactions of 17 lines $(\mathrm{L} 1=$ Han 3475, L33 = 2611, L34 = 953, L39 = Nonglin 9823, L51 = Xinong 8925-13, L53 = Yunfeng 988, L66 = Emai 14, L79 = 298-1, L81 = 3472, L93 = Chen 98-18, L99 = Chuannong 21, L101 = Chuanyu 18, L105 = Kefeng 14, L108 = LB 0458, L122 $=$ Mianyang 29, L132 $=$ SW8588, and L140 = Fengmai 24) were not consistent with those of any known resistance genes. Hence, more work is needed to identify the resistance genes in these lines.
Three lines, L35 (N139), L80 (323-2), and L145 (3S857), conferred resistance to all pathotypes tested, indicating they possess either $\operatorname{Yr} 15$, a gene combination, or one or more unidentified resistance genes.

Slow rusting resistance in field tests. Highly significant differences were found for genotypes and environments for relative AUDPC and FDS in the field trials (Table 3). The genotype-environment interaction was also significant, but its effect 


\begin{tabular}{|c|c|c|c|c|c|c|c|c|c|c|c|c|c|c|}
\hline \multicolumn{15}{|c|}{ Infection types to Puccinia striiformis f. sp. tritici pathotypes } \\
\hline $\begin{array}{c}86036 \\
(\mathrm{P} 12)\end{array}$ & $\begin{array}{c}76088 \\
\text { (P13) }\end{array}$ & $\begin{array}{l}76093 \\
(P 14)\end{array}$ & $\begin{array}{l}86106 \\
(P 15)\end{array}$ & $\begin{array}{c}68009 \\
(\mathbf{P 1 6})\end{array}$ & $\begin{array}{l}86107 \\
\text { (P17) }\end{array}$ & $\begin{array}{l}82061 \\
\text { (P18) }\end{array}$ & $\begin{array}{l}80551 \\
\text { (P19) }\end{array}$ & $\begin{array}{l}85019 \\
\text { (P20) }\end{array}$ & $\begin{array}{l}\text { PE92 } \\
\text { (P21) }\end{array}$ & $\begin{array}{c}\text { CYR26 } \\
\text { (P22) }\end{array}$ & $\begin{array}{c}\text { CYR27 } \\
\text { (P23) }\end{array}$ & $\begin{array}{c}\text { CYR29 } \\
\text { (P24) }\end{array}$ & $\begin{array}{l}\text { CYRSu- } \\
1 \text { (P25) }\end{array}$ & $\begin{array}{c}\text { CYR32 } \\
\text { (P26) }\end{array}$ \\
\hline 0; & 3 & $3+, 4$ & 4 & 4 & 4 & 3 & $2+$ & 4 & $3+, 4$ & $3+$ & 4 & 4 & 3 & 4 \\
\hline 0 & 0 & 0 & 3 & 0,1 & $0 ;, 1$ & 2 & 0 & 2 & 0 & 4 & 3 & 4 & 0 & 4 \\
\hline $0 ;, 1+$ & 3 & $3+, 4$ & $0 ;, 1$ & 0 & 3 & 4 & $2+$ & 3 & 0 & 4 & 4 & 0 & 4 & 4 \\
\hline 0 & 0 & $2,2+$ & 4 & 4 & 4 & $0 ;, 1$ & $2+$ & 0 & 0 & 0 & 4 & 4 & 2 & 4 \\
\hline 0 & $0 ;, 2$ & $0 ;, 2$ & $0 ;, 2$ & 0 & 3 & $0 ;, 1$ & $2+$ & 2 & 3 & $2+$ & 0 & $1+, 2$ & 3 & 4 \\
\hline $0 ;, 2+$ & 0; & 0; & 0; & 2 & 0,1 & 4 & 3 & 3 & 0; & 1,2 & 4 & 4 & 0 & $2+$ \\
\hline 0 & 0; & $1,1+$ & 0; & 2 & 2 & 4 & 3 & 4 & 0; & 4 & 4 & 4 & 1 & 4 \\
\hline 1,2 & 0 & $2,2^{+}$ & $2^{+}$ & 0 & $0 ;, 2$ & 0 & $2,2^{+}$ & $0 ;, 1$ & $0 ;, 1$ & 3 & 4 & $3^{+}, 4$ & 0 & 4 \\
\hline 0; & 4 & 4 & 4 & $3+$ & 4 & 0; & $2+$ & $0 ;, 1$ & 3 & 4 & 0; & 4 & 4 & 4 \\
\hline 0; & $1+2$ & $2+$ & 4 & 4 & 4 & 0; & $1+$ & $0 ;$ & $2,2+$ & $2^{+}$ & $0 ; 1$ & 4 & 3 & $2+$ \\
\hline 0 & 3 & 3 & $0 ;, 2$ & 0 & 3 & 0 & $2^{+}$ & $0 ;, 1$ & $1+, 2$ & 3 & 0 & 4 & 4 & 4 \\
\hline 0 & 0 & 0 & 0 & 0; & 0; & 0; & 0 & 0; & 0 & 0 & 0; & 0 & 0 & $\mathbf{0 , 0}$ \\
\hline 0 & 0 & 0 & 0 & 0 & 0,0 & 0 & 0 & 0 & 0 & 0 & 0 & 0,0 & 0 & 0 \\
\hline 0,0 & 0 & 0 & 0 & 0 & 0 & 0 & 0 & 0 & 0 & 0 & 0 & 0 & 0 & 0,0 \\
\hline 0 & 0 & 0 & 0 & 0 & 0 & 0 & 0 & $0 ; 1$ & 0 & 0 & 0 & 0 & 0 & 0 \\
\hline 0; & 3 & 3 & 0; & 0; & 2 & 0; & 3 & $\mathbf{0}$ & 3 & 3 & 2 & $3+$ & $2+$ & 4 \\
\hline 0,0 & $0,2+$ & $1+$ & $0 ;, 1$ & $0 ;, 1$ & 0 & 0 & $2+$ & 0 & $0 ;, 1$ & 3 & 0 & 0 & 1,2 & 3 \\
\hline 4 & 0; & $1+$ & 4 & 4 & 2 & 4 & 3 & 3 & $\mathbf{0}$ & 0; & 3 & 4 & 2 & 3 \\
\hline 3 & 0 & 2 & 4 & 2 & $0 ;, 2$ & 4 & 3 & 3 & 0 & 1,2 & 4 & 4 & 0 & $2+, 3$ \\
\hline $2+$ & $0 ;, 1$ & 0 & 2 & 2 & $0 ;, 1$ & 3 & $0 ;, 1+$ & 3 & $0 ;, 2$ & 0 & 3 & $3+, 4$ & 0 & $0 ; 1+$ \\
\hline $2+$ & $0 ; 1$ & $0 ; 1+$ & 0,0 & 3 & $0 ; 1$ & 3 & $2+$ & $3+$ & $0 ; 1$ & 0 & 0 & 0 & $0 ;, 1$ & $1+, 2$ \\
\hline 0; & 0 & 4 & $3+$ & 4 & 0 & 0; & 4 & 4 & $\mathbf{0 , 0}$ & 4 & 4 & 4 & 4 & 4 \\
\hline 0; & 0; & 0; & 0; & 0; & 1 & 4 & 1,2 & 4 & 0; & 0; & 0; & 3 & 0; & 3 \\
\hline 0; & $3+$ & 4 & 3 & 0 & 3 & 0; & 4 & o & 4 & 0; & 3 & 0; & 3 & $0 ;, 1$ \\
\hline $\mathbf{0 , 0}$ & 0 & 0 & 0 & 0 & 0 & 0 & 0 & 0 & 0; & 0 & 0; & 0 & 0 & 0 \\
\hline 0, 0 & 0 & $2+$ & 2 & 0; & 0; & 0; & 3 & 0; & $0 ;, 1+$ & 0; & 0; & 4 & 2 & 4 \\
\hline $0 ;, 1$ & $2+$ & $1+$ & $0 ;, 2$ & $2+$ & 4 & 2 & $2+$ & 2 & 4 & 4 & 4 & 4 & 0; & 4 \\
\hline $0 ; 1+$ & 0,0 & 0; & 3,4 & 0; & 2 & 0; & 2 & 3 & $2+$ & 4 & 4 & 4 & 1 & $3+$ \\
\hline 0; & 0; & 0; & 0; & 3 & $\overline{1}$ & 4 & $0 ;, 1$ & 4 & 0; & 0; & 2 & 4 & 0; & 3 \\
\hline 0; & 0; & 3 & 4 & 4 & $2+$ & 3 & $0 ;, 1+$ & 4 & 3 & 0; & 4 & 4 & 2 & $2+$ \\
\hline- & 0 & - & o & 0 & 0; & - & 3 & o & 0; & 0 & - & 4 & 0 & 3 \\
\hline 0; & $\mathbf{0 , 0}$ & 0 & $2+$ & 0; & 0; & 3 & 0; & 3 & $\mathbf{0 , 0}$ & 0 & 4 & o & 0 & 4 \\
\hline 0 & 0; & $2+$ & 0; & 0 & 0 & 3 & 3 & 0 & 0 & 0,2 & 0 & 0; & 4 & 4 \\
\hline 3 & 0 & 0 & 3 & 4 & 3 & 3 & 4 & 0 & 3 & 4 & 4 & 0 & 1,2 & 4 \\
\hline 3,4 & 0 & $2+$ & 3 & 0 & 3 & 4 & 3 & 0 & 3,3 & 0 & 3 & 4 & 4 & 4 \\
\hline 0 & 0,0 & $0: 2$ & 0 & 0 & 0 & 4 & 0 & 3 & 0 & 0 & 4 & 4 & 0 & 4 \\
\hline $0 ;, 1$ & $0 ;, 1$ & $0 ;, 1$ & 1,2 & 0 & 3 & 0 & 4 & 3 & 3 & 4 & 3 & $2+$ & 1,2 & 3 \\
\hline 3 & 4 & 3 & 4 & 4 & 4 & 4 & 4 & 4 & 4 & 4 & 4 & 3 & 3 & 4 \\
\hline 3 & 3 & 2 & 3 & 3 & 3 & 4 & 3 & 3 & 3 & 4 & 3 & 4 & 3 & 4 \\
\hline 4 & $2^{+}$ & 3,4 & 4 & 3 & 3 & 3 & 4 & 4 & 4 & 3 & 4 & 3 & 3 & 4 \\
\hline 0 & 0,0 & 0,0 & 3 & 2 & 4 & 4 & 4 & 4 & 4 & 4 & 4 & 3,4 & 1,2 & 4 \\
\hline 4 & 4 & 4 & 4 & 4 & 4 & 4 & 4 & 4 & 4 & 4 & 4 & 4 & 4 & 4 \\
\hline 4 & 3 & 4 & 4 & 4 & 4 & 4 & 4 & 4 & 4 & 4 & 4 & 4 & 4 & 4 \\
\hline 4 & 3 & 3 & 4 & 3 & 4 & 4 & 4 & 4 & 4 & 3 & 4 & 4 & 3 & 4 \\
\hline 0,0 & 3 & 0 & 0 & 0 & 3 & 1 & $3,3+$ & 4 & $0 ;, 1$ & 3 & 3 & $3+$ & 3 & 4 \\
\hline 3 & 0 & 3 & 0 & 1,2 & 3 & 4 & 4 & 4 & 0 & 0 & 3 & 3,4 & 4 & 4 \\
\hline 4 & 3 & 4 & 4 & 3 & 3 & 3 & 4 & 4 & 4 & 4 & 4 & 3 & 4 & 3 \\
\hline 3 & 3 & $2^{+}$ & 0,1 & 3 & 3 & 4 & 4 & 4 & 4 & 4 & 4 & 4 & 3 & 4 \\
\hline 4 & 3 & 0 & 1,2 & 1,2 & $0 ;, 1$ & 1 & 4 & 3 & 4 & 0 & 3 & 4 & 4 & 3 \\
\hline 3 & 3 & 0 & $0 ;, 1$ & $2+$ & 3 & $0 ;, 1$ & 4 & 3 & 4 & 0 & 2 & 4 & 3 & 4 \\
\hline 4 & 4 & 4 & 3 & $3+$ & 4 & 3 & 4 & 4 & 4 & 4 & 3 & 4 & 3 & 4 \\
\hline 4 & 4 & 4 & 4 & 4 & 3 & 4 & 4 & 4 & 0 & $0 ;, 1$ & 4 & 4 & 4 & 4 \\
\hline 3 & 3 & 4 & $0 ;, 1$ & 3 & 2 & 0 & 3 & 3 & 4 & 2 & 3 & 4 & $0 ; 2$ & 3 \\
\hline 3 & 1,2 & 4 & $0 ;, 2$ & 3 & 4 & 3 & 4 & 4 & 3 & 4 & 4 & 3 & 3 & 3 \\
\hline 0,0 & 0 & 0 & 0 & 0 & 0,0 & 0 & $0 ;, 1+$ & 0 & 0 & 0 & 0 & $3+$ & 0 & 4 \\
\hline 3 & 2 & 3 & $0 ;, 1$ & 0 & 3 & 3 & 4 & 4 & 3 & 2 & 4 & 3 & 4 & 4 \\
\hline
\end{tabular}

on variation was much lower than that for genotype differences.

FDS of susceptible check, Tiaogan 601, was 90 to 100 in Beijing and Tianshui (Table 4), indicating disease developed well at both locations. The FDS of the slow rusting check, Lantian 12, was 13 and 19, respectively, while its relative AUDPC was 0.17 and 0.16 , respectively, at the two locations (Table 4).

Twenty-six lines, including L23 (Zhoumai 11), L55 (93-23-13), L57 (95-108),
L58 (95-111-6-3-10), L60 (95-3-5-1), L61 (9562-1), L72 (An 96-8), L77 (01-3570), L80 (323-2), L83 (58512-2), L90 (99-607), L94 (Chuanmai 42), L95 (Chuanmai 43), L104 (Jinfeng 626), L107 (LB 0438), L109 (Lemai 3), L113 (Mian 2000-34), L115 (Mian 2000-8), L117 (Mianmai 37), L125 (Nei 2938), L126 (Neimai 9), L127 (R111), L128 (R122), L129 (R25), L134 (Xike 01015), and L136 (Xingmai 2), were identified as highly resistant cultivars with high resistance to the pathotype CYR32 at both seedling and adult stages. Thirty-three lines (Table 4) showed slow rusting resistance at two locations, exhibiting high infection types at seedling stage, but low FDS and AUDPC in the field tests.

The correlations of FDS and relative AUDPC between Beijing and Tianshui were 0.88 and 0.80 , respectively, indicating a high consistency of the test in two locations for slow rusting resistance. The correlation between relative AUDPC and FDS at the two locations varied from 0.91 
to 0.98 , indicating that FDS could be used as a reliable indicator of AUDPC.

\section{DISCUSSION}

Seedling test for stripe rust. In the present study, $\operatorname{Yr} 9$ was identified in 42 of 98 $(43 \%)$ lines, which is consistent with the results reported by Niu et al. (21) and $\mathrm{Wu}$ et al. (43). Yr26 was postulated to be present in 19 lines. This gene was derived from Chinese line $\gamma 80-1$ (Triticum turgidum L.) and its derivatives such as
92R137, 92R171, and 92R178 from Nanjing Agricultural University (Table 1) (16). The lines possessing $\operatorname{Yr} 26$ are grown over a large area in Gansu and Sichuan provinces where climatic conditions are favorable to stripe rust development. If a new race of $P$. striiformis $\mathrm{f}$. sp. tritici virulent to Yr26 appears, a new epidemic could again cause severe losses as the case in 1990 and with the Fan 6-derived resistance in 2002. The danger of using limited sources of resistance has become a great concern to

Table 3. Analysis of variance of relative area under the disease progress curve (AUDPC) and final disease severity (FDS) in 137 wheat lines including slow rusting and susceptible checks tested in Beijing in 2003-2004, and in Tianshui in 2003-2004 and 2004-2005 cropping seasons

\begin{tabular}{llrrrc}
\hline Parameter & $\begin{array}{l}\text { Source of } \\
\text { variation }\end{array}$ & df & MS & $\boldsymbol{F}$ value & $\boldsymbol{P} \boldsymbol{F}$ \\
\hline AUDPC & Line & 136 & 0.92 & 106.89 & $<0.0001$ \\
& Env & 2 & 1.36 & 157.51 & $<0.0001$ \\
& Replicate & 6 & 0.0094 & 1.10 & 0.36 \\
& Lines $\times$ env & 272 & 0.062 & 7.15 & $<0.0001$ \\
FDS & Error & 816 & 0.0086 & & $<0.0001$ \\
& Line & 136 & $9,972.99$ & 207.46 & $<0.0001$ \\
& Env & 2 & $13,181.77$ & 274.21 & $<0.0001$ \\
& Replicate & 6 & 35.90 & 8.67 & $<0.000$ \\
\hline
\end{tabular}

wheat breeders and pathologists. It is imperative that new stripe rust resistance genes be identified and utilized in breeding programs. Although $\mathrm{Yr} 26$ currently provides effective resistance in China, breeding programs should anticipate its downfall by improving the genetic diversity of breeding materials.

The Yr26-virulent race $(\mathrm{P} 4=75078)$ originated from Egypt (22). Apart from its Yr26 virulence, it is probably not a threatening pathotype. This culture was also virulent on the line $Y r 24 / 3 *$ Avocet S, indicating that $Y r 26$ and $Y r 24$ are likely the same gene, which is in agreement with our previous study (10). Although $Y r 24$ was also derived from T. turgidum (19), its origin and naming was independent of that for Yr26.

Near-isogenic lines (NIL) with one resistance gene were often utilized in the postulation of resistance genes to wheat leaf rust $(23,34)$. Nevertheless, in the postulation of stripe rust resistance genes, differential lines with one or more genes were often employed $(24,26)$. In the present study, 8 of 25 differential lines contain more than one resistance gene, leading to some difficulties in gene postulation in

Table 4. Wheat lines with slow rusting resistance to stripe rust identified by using Fisher's $F$ protected LSD (LSD alpha $=0.05)$ test in Beijing in 20032004, and in Tianshui in 2003-2004 and 2004-2005 cropping seasons

\begin{tabular}{|c|c|c|c|c|c|c|c|c|c|c|}
\hline \multirow[b]{2}{*}{ Line number } & \multirow{2}{*}{$\begin{array}{c}\text { Seedling IT } \\
\text { to CYR32 }\end{array}$} & \multicolumn{3}{|c|}{ Beijing (2003-2004) } & \multicolumn{3}{|c|}{ Tianshui (2003-2004) } & \multicolumn{3}{|c|}{ Tianshui (2004-2005) } \\
\hline & & IT & FDS & rAUDPC & IT & FDS & rAUDPC & IT & FDS & rAUDPC \\
\hline Lantian $12^{\mathrm{a}}$ & 4 & 1,2 & 13 & 0.17 & 1,2 & 17 & 0.14 & 3 & 21 & 0.18 \\
\hline Tiaogan $601^{\mathrm{b}}$ & 4 & 4 & 90 & 1 & 4 & 95 & 1 & 4 & 100 & 1 \\
\hline Lankao 906-4 (L9) & 4 & 3,4 & 25 & 0.24 & 3,4 & 12 & 0.12 & 4 & 12 & 0.1 \\
\hline Xinong 291 (L50) & 4 & 0 & 1 & 0.01 & 2 & 1 & 0.01 & $0 ;, 2$ & 1 & 0.01 \\
\hline Shaannong 981 (L44) & 4 & 2,3 & 12 & 0.19 & 2,3 & 15 & 0.1 & 2,3 & 25 & 0.21 \\
\hline 90-99-5 (L54) & 4 & 3,4 & 3 & 0.11 & 3,4 & 7 & 0.09 & 4 & 17 & 0.21 \\
\hline $94 \mathrm{t}-133$ (L56) & $3+$ & 0 & 1 & 0.01 & 1,2 & 3 & 0.06 & 1,2 & 2 & 0.07 \\
\hline $96-22-2-3$ (L62) & 4 & 1,2 & 6 & 0.07 & 2,3 & 10 & 0.07 & 2,3 & 6 & 0.09 \\
\hline Lantian 9 (L63) & 4 & 2,3 & 6 & 0.06 & $0 ;, 1$ & 1 & 0.02 & 1,2 & 5 & 0.1 \\
\hline Guizhou 98-18 (L74) & 3 & 2,3 & 6 & 0.15 & 3,4 & 24 & 0.15 & 3,4 & 24 & 0.25 \\
\hline 01-018 (L78) & 3 & 1,2 & 4 & 0.1 & $3,3+$ & 15 & 0.14 & 3,4 & 1 & 0.05 \\
\hline 01-10251 (L76) & 4 & 1,2 & 19 & 0.25 & 2,3 & 16 & 0.13 & 2,3 & 8 & 0.1 \\
\hline 351-15 (L82) & 4 & 1 & 2 & 0.02 & 2 & 2 & 0.03 & 2 & 11 & 0.12 \\
\hline 58749 (L84) & 4 & 3,4 & 9 & 0.11 & 3,4 & 4 & 0.04 & 3,4 & 4 & 0.1 \\
\hline 93Xia032-1 (L86) & 3 & $3,3+$ & 18 & 0.21 & $3,3+$ & 8 & 0.08 & $3,3+$ & 7 & 0.11 \\
\hline 99422 (L89) & 3 & 2,3 & 16 & 0.2 & 2,3 & 4 & 0.07 & 2,3 & 4 & 0.07 \\
\hline Bi 2002-2 (L73) & 3 & 2,3 & 14 & 0.1 & 3 & 8 & 0.07 & 3 & 12 & 0.11 \\
\hline Chen 98-18 (L93) & 4 & 2,3 & 16 & 0.26 & 2,3 & 22 & 0.23 & 2 & 24 & 0.23 \\
\hline Chuannong 17 (L98) & 3 & 1,2 & 11 & 0.11 & 2,3 & 12 & 0.12 & 2,3 & 11 & 0.14 \\
\hline Chuannong 21 (L99) & 3 & 1,2 & 5 & 0.09 & 2,3 & 12 & 0.11 & 3,4 & 15 & 0.14 \\
\hline Chuanyu 18 (L101) & 4 & 1,2 & 1 & 0.1 & 3 & 2 & 0.03 & $3+$ & 2 & 0.02 \\
\hline Chuanyu 19 (L102) & 4 & 3,4 & 8 & 0.14 & 3,4 & 5 & 0.06 & 4 & 4 & 0.11 \\
\hline LB 0288 (L106) & 4 & 1,2 & 15 & 0.07 & 2,3 & 3 & 0.04 & 2,3 & 14 & 0.11 \\
\hline LB 0458 (L108) & 3 & 1,2 & 7 & 0.05 & 1,2 & 4 & 0.05 & 2 & 12 & 0.1 \\
\hline Mian 2000-13 (L111) & 3 & 1,2 & 9 & 0.18 & 3 & 12 & 0.08 & 3,4 & 7 & 0.1 \\
\hline Mian 2000-18 (L112) & 3 & 1 & 1 & 0.01 & 2 & 1 & 0.02 & 2,3 & 2 & 0.06 \\
\hline Mian 2000-36 (L114) & 4 & 1,2 & 2 & 0.04 & 3,4 & 9 & 0.09 & 3,4 & 21 & 0.2 \\
\hline N1621-19-4 (L123) & 3 & 1,2 & 2 & 0.16 & 2 & 1 & 0.03 & 3,4 & 5 & 0.11 \\
\hline N711 (L124) & 4 & 0 & 5 & 0.01 & 1,2 & 1 & 0.03 & 2,3 & 2 & 0.07 \\
\hline R88 (L130) & 4 & 0 & 1 & 0.01 & 3 & 2 & 0.02 & 3,4 & 1 & 0.03 \\
\hline R97 (L131) & 4 & 3 & 1 & 0.09 & 3 & 9 & 0.08 & 1,2 & 2 & 0.06 \\
\hline SW 8588 (L132) & 4 & 0 & 1 & 0.01 & $2+$ & 1 & 0.01 & 3 & 3 & 0.03 \\
\hline 9021 (L139) & 3 & $0 ; 1$ & 5 & 0.02 & 2,3 & 22 & 0.19 & 2,3 & 11 & 0.15 \\
\hline Jing 035-1 (L141) & 4 & $0 ;, 1$ & 11 & 0.19 & $0 ;, 2$ & 21 & 0.21 & 2,3 & 21 & 0.15 \\
\hline Yin 11-12 (L143) & 4 & 3 & 3 & 0.04 & 2,3 & 6 & 0.09 & 3 & 12 & 0.15 \\
\hline $\operatorname{LSD}(0.05)$ & & & 13.01 & 0.21 & & 10.73 & 0.12 & & 9.40 & 0.10 \\
\hline
\end{tabular}

\footnotetext{
a Slow rusting check.
}

b Susceptible check. 
genetically unknown materials. Some gene combinations may be identified, but others cannot be postulated due to a lack of testers with confirmed single resistance genes. The near-isogenic lines developed in Avocet $\mathrm{S}$ may be a good option for reference lines (39), but the series including limited known genes needs to be extended to cover a wider range of genes. The differential lines used in this study are all with universally known genes (20). Most of them contain only one gene, assuring the postulation of resistance genes. In addition, the most frequent resistance gene, $\mathrm{Yr} 9$, identified in the seedling test was confirmed by a SCAR marker of 1B/1R (Table 1), and the pedigree information was also used in the postulation of seedling resistance genes.

In the seedling test for stripe rust, it very likely causes confusion with IT 0 , confusing true immune response (low) and escape (missing data) with the other response that infection has occurred but variability between tests can still be a problem. In the present study, the seedling tests were conducted twice, and the results were consistent between two seedling tests with very few exceptions. In case disease scores with some pathotypes tested were not consistent between two repeats, the data with better disease development were used for the postulation of seedling resistance genes, because the low infection types might be due to inadequate inoculation.

Field resistance. Of 33 lines with slow rusting resistance to CYR32, 26 were tested on seedlings with 26 pathotypes; 15 lines carried $\operatorname{Yr} 9$ and 6 lines gave high ITs to most of the pathotypes (Table 2). In these lines, the major seedling resistance genes were overcome by pathotype CYR32, and the slow rusting resistances probably resulted from minor genes. The resistance in 96-22-2-3 (L62) may be conferred by $\mathrm{Yr} 16$ derived from Cappelle Desprez (Table 1) (41). Slow rusting in 94t133 (L56) may be derived from Xiannong 4 with durable resistance to stripe rust (Table 1) (46). Slow rusting resistance in Chuanyu 18 (L101), Chuanyu 19 (L102), LB 0288 (L106), LB 0458 (L108), 9021 (L139), and Yin 11-12 (L143) may be derived from CIMMYT wheat germ plasm (Table 1). All lines except for Lankao 9064 (L9) with slow rusting resistance identified in this study were from northwestern and southwestern China where stripe rust resistance is a major breeding objective, indicating that more attention was paid to wheat stripe rust resistance in western China than in other wheat growing regions.

Breeding for durable resistance with seedling and slow rusting genes. In China, urediospores of $P$. striiformis $\mathrm{f}$. sp. tritici live over summer in southeastern Gansu and northeastern Sichuan, where climatic conditions, geographic characteristics, and cropping systems are favorable for stripe rust every year (11). The spores spread from west to east in the autumn, winter, and early spring, and result in epidemics of stripe rust in the main wheat growing region of China. Therefore, reducing the amount of initial inoculum in the over-summer regions is a key strategy to achieving sustainable control of stripe rust in China (35). Several strategies, such as pyramiding of major resistance genes, deployment of different genes in mountainous regions (where urediospores can live in summer) and plains (over-winter region), and reducing wheat acreage by planting other crops, can largely reduce inoculum in the over-summer regions $(35,42)$. In the present study, 26 lines with good agronomic performance were found to be highly resistant to stripe rust both in the seedling and adult plant stage with different resistance genes, which may be used as parents in the wheat breeding program for gene deployment and gene pyramiding in southeastern Gansu and northeastern Sichuan to reduce the amount of urediospores of $P$. striiformis f. sp. tritici. This will certainly help in the control of stripe rust in other wheat growing regions.

The eastern and northern epidemic region covers major wheat production areas with less frequent occurrence of favorable climatic conditions, especially in Hebei, Henan, Shandong, and Shanxi provinces (35). Strategies for controlling stripe rust in these regions are slightly different from those in the over-summer regions of western China. In addition to pyramiding and deploying major resistance genes, utilization of minor additive genes was strongly proposed for controlling stripe rust (21). During the past 5 years, a shuttle-breeding program was conducted between CIMMYT and China for developing slow rusting cultivars with a limited backcrossing method (31). CIMMYT wheat cultivars Chapio, Tukuru, Kukuna, and Vivitsi, with four to five minor additive genes, were used as parents in wheat breeding. In this study, six lines, including Chuanyu 18 (L101), Chuanyu 19 (L102), LB 0288 (L106), LB 0458 (L108), 9021 (L139), and Yin 11-12 (L143), were identified as having slow rusting resistance, which was derived from the CIMMYT wheat germ plasm, indicating that it is possible to combine slow rusting resistance with good agronomic performance through shuttle breeding between Mexico and China. Because multiple genes may confer durable resistance $(27,31)$, the use of slow rusting resistance should be a more sustainable strategy for developing wheat cultivars in eastern and northern China.

In the evaluation of adult-plant resistance (APR) to wheat rusts, AUDPC is certainly a more reliable indicator than FDS. However, in the wheat breeding program, breeders need to evaluate the disease severity for hundreds of thousands of individuals and thousands of lines. They may have no time to score the disease several times to calculate AUDPC. A simple method is therefore needed for evaluating such a great number of materials. In the present study, a high correlation was found between AUDPC and FDS, which is consistent with our previous studies in the characterization of APR to powdery mildew in wheat $(12,38)$. Hence, we suggested using FDS as an indicator for the evaluation of APR to stripe rust in wheat breeding with a single scoring at an appropriate time. The high correlations of FDS and AUDPC between Beijing and Tianshui indicated a high degree of repeatability between sites and seasons. The Tianshui environment with low temperature and much more rain is very favorable to the development of stripe rust and for selection of slow rusting resistance in wheat breeding populations.

\section{ACKNOWLEDGMENTS}

The authors are very grateful to R. A. McIntosh for reviewing the manuscript. This project was funded by the National 863 program (2003AA207090) and National Natural Science Foundation of China (30220140636 and 30471083).

\section{LITERATURE CITED}

1. Bariana, H. S., and McIntosh, R. A. 1993. Cytogenetic studies in wheat XV. Location of rust resistance genes in VPM1 and their genetic linkage with other disease resistance genes in chromosome 2A. Genome 36:476482.

2. Bartos, P., Johnson, R., and Stubbs, R. W. 1987. Postulated genes for resistance to yellow rust in Czechoslovakian wheat cultivars. Cereal Rusts Bull. 15:79-84.

3. Broers, L. H. M., Cuesta Subias, X., and Lopez Atilano, R. M. 1996. Field assessment of quantitative resistance to yellow rust in ten spring bread wheat cultivars. Euphytica 90:916.

4. Caldwell, R. M. 1968. Breeding for general and/or specific plant disease resistance. Pages 263-272 in: Proc. 3rd Int. Wheat Genetics Sympos. K. W. Findlay and K. W. Shepherd, eds. Australian Acad. Sci., Canberra, Australia.

5. Chen, X. M., and Line, R. F. 1995. Gene action in wheat cultivars for durable, hightemperature, adult-plant resistance and interaction with race-specific, seedling resistance to Puccinia striiformis. Phytopathology 85:567572.

6. Chen, X. M., and Line, R. F. 1995. Gene number and heritability of wheat cultivars with durable, high-temperature, adult-plant (HTAP) resistance and interaction of HTAP and racespecific seedling resistance to Puccinia striiformis. Phytopathology 85:573-578.

7. Flor, H. H. 1955. Host parasite interaction in flax rust: Its genetics and implication. Phytopathology 45:680-685.

8. Fransis, H. A., Leitch, A. R., and Koebner, R. M. D. 1995. Conversion of a RAPD-generated PCR product, containing a novel dispersed repetitive element, into a fast robust assay for the presence of rye chromatin in wheat. Theor. Appl. Genet. 90:636-642.

9. He, Z. H., Rajaram, S, Xin, Z. Y., and Zhang, G. Z., eds. 2001. A History of Wheat Breeding in China. CIMMYT, Mexico, D.F.

10. Li, G. Q., Li, Z. F., Yang, W. Y., Zhang, Y., He, Z. H., Xu, S. C., Singh, R. P., Qu, Y. Y., and Xia, X. C. 2006. Molecular mapping of stripe rust resistance gene $\mathrm{YrCH} 42$ in Chinese wheat cultivar Chuanmai 42 and its allelism 
with $Y r 24$ and $Y r 26$. Theor. Appl. Genet. 112:1434-1440.

11. Li, Z. Q., and Zeng, S. M. 2000. Wheat Rusts in China. China Agriculture Press, Beijing.

12. Liang, S. S., Suenaga, K., He, Z. H., Wang, Z. L., Liu, H. Y., Wang, D. S., Singh, R. P., Sourdille, P., and Xia, X. C. Quantitative trait loci mapping for adult-plant resistance to powdery mildew in bread wheat. Phytopathology 96:784-789.

13. Line, R. F., and Chen, X. M. 1995. Successes in breeding for and managing durable resistance to wheat rusts. Plant Dis. 79:1254-1255.

14. Loegering, W. Q., and Burton, C. H. 1974. Computer-generated hypothetical genotypes for reaction and pathogenicity of wheat cultivars and cultures of Puccinia graminis tritici. Phytopathology 64:1380-1384.

15. Ma, H., and Singh, R. P. 1996. Expression of adult resistance to stripe rust at different growth stages of wheat. Plant Dis. 80:375-379.

16. Ma, J. X., Zhou, R. H., Dong, Y. S., Wang, L. F., Wang, X. M., and Jia, J. Z. 2001. Molecular mapping and detection of the yellow rust resistance gene $Y r 26$ in wheat transferred from Triticum turgidum L. using microsatellite markers. Euphytica 120:219-226.

17. Martinez, F., Niks, R. E., and Rubiales, D. 2001. Partial resistance to leaf rust in a collection of ancient Spanish barleys. Hereditas 135:199-203.

18. McIntosh, R. A. 1992. Close genetic linkage of genes confering adult-plant resistance to leaf rust and stripe rust in wheat. Plant Pathol. 41:523-527.

19. McIntosh, R. A., and Lagudah, E. S. 2000. Cytogenetical studies in wheat XVIII. Gene Yr24 for resistance to stripe rust. Plant Breed. 119:81-83.

20. McIntosh, R. A., Yamazaki, Y., Devos, K. M., Dubcovsky, J., Rogers, J., and Appels, R. 2003. Catalogue of gene symbols for wheat. Proc. 10th Int. Wheat Genet. Sympos., Vol. 1, Instituto Sperimentale per la Cerealcoltura, Rome Italy.

21. Niu, Y. C., Qiao, Q., and Wu, L. R. 2000. Postulation of resistance genes to stripe rust in commercial wheat cultivars from Henan, Shandong, and Anhui provinces. Acta Phytopathol. Sinica 30:122-128.

22. Niu, Y. C., and Wu, L. R. 1997. The breakdown of resistance to stripe rust in Fan 6 and Mianyang wheat cultivars and strategies for its control. Acta Phytopathol. Sinica 27:5-8.

23. Oelke, L. M., and Kolmer, J. A. Characterization of leaf rust resistance in hard red spring wheat cultivars. Plant Dis. 88:1127-1133.

24. Perwaiz, M. S., and Johnson, R. 1986. Genes for resistance to yellow rust in seedlings of wheat cultivars from Pakistan tested with British isolates of Puccinia striiformis. Plant Breed 97:289-296.

25. Peterson, R. F., Campbell, A. B., and Hannah, A. E. 1948. A diagrammatic scale for estimatng rust intensity of leaves and stems of cereals. Can. J. Res. 26:496-500.

26. Sharma, S., Louwers, J. M., Karki, C. B., and Snijders, C. H. A. 1995. Postulation of resistance genes to yellow rust in wild emmer wheat derivatives and advanced wheat lines from Nepal. Euphytica 81:271-277.

27. Sharp, E. L., and Volin, R. B. 1970. Additive genes in wheat conditioning resistance to stripe rust. Phytopathology 60:1146-1147.

28. Singh, H., Johnson, R., and Seth, D. 1990. Genes for race-specific resistance to yellow rust (Puccinia striiformis) in Indian wheat cultivars. Plant Pathol. 39:424-433.

29. Singh, R. P. 1992. Genetic association of leaf rust resistance gene $\mathrm{Lr} 34$ with adult plant resistance to stripe rust in bread wheat. Phytopathology 82:835-838.

30. Singh, R. P. 1993. Genetic association of gene $B d v 1$ for tolerance to barley yellow dwarf virus with genes $L r 34$ and $\mathrm{Yr} 18$ for adult plant resistance to rusts in bread wheat. Plant Dis. 77:1103-1106.

31. Singh, R. P., Huerta-Espino, J., and Rajaram, S. 2000. Achieving near-immunity to leaf and stripe rusts in wheat by combining slow rusting resistance genes. Acta Phytopathol. Hung. 35:133-139.

32. Singh, R. P., and Rajaram, S. 1994. Genetics of adult plant resistance to stripe rust in ten bread wheats. Euphytica 72:1-7.

33. Suenaga, K., Singh, R. P., Huerta-Espino, J., and William, H. M. 2003. Microsatellite markers for genes $\mathrm{Lr} 34 / \mathrm{Yrl}$ a and other quantitative trait loci for leaf rust and stripe rust resistance in bread wheat. Phytopathology 93:881-890.

34. Wamishe, Y. A., and Milus, E. A. 2004. Seedling resistance genes to leaf rust in soft red winter wheat. Plant Dis. 88:136-146.

35. Wan, A., Zhao, Z., Chen, X., He, Z., Jin, S., Jia, Q., Yao, G., Yang, J., Wang, B., Li, G., Bi, Y., and Yuan, Z. 2004. Wheat stripe rust epidemic and virulence of Puccinia striiformis $\mathrm{f}$. sp. tritici. Plant Dis. 88:896-904.

36. Wang, F. L., Wu, L. R., Wan, A. M., Song, W. Z., Yuan, W. H., and Yang, J. X. 1994. Postulated genes for resistance to stripe rust in seedlings of wheat cultivars from Shaanxi, Gansu and Sichuan provinces. Acta Agron. Sinica 20:589-594.

37. Wang, F. L., Wu, L. R., Xie, S. X., and Wan, A. M. 1994. Postulation of genes and adult resis- tance to stripe rust of Chinese important wheat resistance resources. Acta Phytopathol. Sinica 24:175-180.

38. Wang, Z. L., Li, L. H., He, Z. H., Duan, X. Y Zhou, Y. L., Chen, X. M., Lillemo, M., Singh, R. P., Wang, H., and Xia, X. C. 2005. Seedling and adult plant resistance to powdery mildew in Chinese bread wheat cultivars and lines. Plant Dis. 89:457-463.

39. Wellings, C. R., Singh, R. P., McIntosh, R. A., and Pretorius, Z. A. 2004. The development and application of near isogenic lines for the wheat stripe (yellow) rust pathosystem. 11th Int. Cereal Rusts Powdery Mildews Conf. John Innes Centre, Norwich, England, 22nd to 27th August 2004. A1.39.

40. William, M., Singh, R. P., Huerta-Espino, J., Ortiz Islas, S., and Hoisington, D. 2003. Molecular marker mapping of leaf rust resistance gene $L r 46$ and its association with stripe rust resistance gene $Y r 29$ in wheat. Phytopathology 93:153-159.

41. Worland, A. J., and Law, C. N. 1986. Genetic analysis of chromosome 2D of wheat. I. The location of genes affecting height, day-length insensitivity, hybrid dwarfism and yellowrust resistance. Z. Pflanzenzüchtg 96:331 345.

42. Wu, L. R., Wan, A. M., Niu, Y. C., Jin, S. L. and Li, J. P. 1999. Integrated pest management on wheat in the Longnan region. Pages 34-38 in: Research Progress in Plant Protection and Plant Nutrition. China Association of Agricultural Science Societies, China Agricultural Press, Beijing, China.

43. Wu, L. R., Yang, H. A., Yuan, W. H., Song, W. Z., Yang, J. X., Li, Y. F., and Bi, Y. Q. 1993. On the physiological specialization of stripe rust of wheat in China during 1985-1990. Acta Phytopathol. Sinica 23:269-274.

44. Yang, Z. M., Xie, C. J., and Sun, Q. X. 2003. Situation of the sources of stripe rust resistance of wheat in the Post-CY32 Era in China. Acta Agron. Sinica 29:161-168.

45. Zadoks, J. C. 1961. Yellow rust on wheat: Studies on epidemiology and physiological specialization. Tijdschr. Plantenziekten T. Pl. Ziekten 67:69-256.

46. Zhang, Z. J., Yang, G. H., Li, G. H., Jin, S. L., and Yang, X. B. 2001. Transgressive segregation, heritability, and number of genes controlling durable resistance to stripe rust in one Chinese and two Italian wheat cultivars. Phytopathology 91:680-686.

47. Zhou, X. C. 2003. Strategies and research methodologies for a sustainable genetic control of wheat stripe rust in China. Petria 13:125 138. 